ESAIM: M2AN 55 (2021) 2445-2472

https://doi.org/10.1051/m2an/2021053
ESAIM: Mathematical Modelling and Numerical Analysis

www.esaim-m2an.org

\title{
FAST SOLVER FOR QUASI-PERIODIC 2D-HELMHOLTZ SCATTERING IN LAYERED MEDIA
}

\author{
José Pinto®, Ruben Aylwin@ and Carlos Jerez-Hanckes*i]
}

\begin{abstract}
We present a fast spectral Galerkin scheme for the discretization of boundary integral equations arising from two-dimensional Helmholtz transmission problems in multi-layered periodic structures or gratings. Employing suitably parametrized Fourier basis and excluding cut-off frequencies (also known as Rayleigh-Wood frequencies), we rigorously establish the well-posedness of both continuous and discrete problems, and prove super-algebraic error convergence rates for the proposed scheme. Through several numerical examples, we confirm our findings and show performances competitive to those attained via Nyström methods.
\end{abstract}

Mathematics Subject Classification. 65N35, 65N38, 45M15, 78A45.

Received September 30, 2020. Accepted August 30, 2021.

\section{INTRODUCTION}

A vast number of scientific and engineering applications rely on harnessing acoustic and electromagnetic wave diffraction by periodic and/or multilayered domains. Current highly demanding operation conditions for such devices require solving thousands of specific settings for design optimization or the quantification of shape or parameter uncertainties in the relevant quantities of interest, challenging the scientific computing community to continuously develop ever more efficient, fast and robust solvers $(c f .[8,18,31,39,40]$ and references therein). Assuming impinging time-harmonic plane waves, scattered and transmitted fields have been solved by a myriad of mathematical formulations and associated solution schemes. These range from volume variational formulations to various boundary integral representations and equations $(c f .[2,3,9,11,21,33])$, pure or coupled implementations of finite and boundary element methods ( $c f .[3,4,22,34,40]$ or [36], Chap. 5) and Nyström methods $[13,16,19,24,30]$.

In this work, we build upon our theoretical review [5] and present a spectral Galerkin method for solving second-kind direct boundary integral equations (BIEs) for the Helmholtz transmission problem for twodimensional, periodic multi-layered gratings with smooth interfaces. Contrary to the low-order local basis functions used in the standard boundary element method, spectral bases are composed of high-order polynomials whose support lie on the whole scatterer boundary or on large portions of it. Successfully employed on two- and three-dimensional scattering problems $[23,25,26]$, the main advantage of a spectral discretization is the ability to converge at a super-algebraic rate whenever solutions are smooth enough. Hence, our proposed

Keywords and phrases. Boundary integral equations, quasi-periodic scattering, spectral elements, gratings, multi-layered domain.

Faculty of Engineering and Sciences, Universidad Adolfo Ibáñez, Santiago, Chile.

*Corresponding author: carlos.jerez@uai.cl

(C) The authors. Published by EDP Sciences, SMAI 2021

This is an Open Access article distributed under the terms of the Creative Commons Attribution License (https://creativecommons.org/licenses/by/4.0), which permits unrestricted use, distribution, and reproduction in any medium, provided the original work is properly cited. 
method can in practice compete with Nyström methods while simultaneously inheriting all of the theoretical aspects of classical Galerkin methods.

In two dimensions, spectral methods are closely related to the theory of periodic pseudo-differential operators [37], since the discretization through spectral elements can be interpreted as a truncation of the associated Fourier series where the action of the operators is well understood. We show that wave scattering by periodic domains is closely connected to the bounded domain case, making it possible to reuse almost all the pseudodifferential operator theory for our analysis. Key to our analysis are the results in $[22,34,41]$ regarding the unique solvability and eigenvalues of the associated volume problem. From here, we deduce that our BIE is uniquely solvable except at a countable set of wavenumbers composed of cut-off frequencies - wavenumbers for which the series defining the quasi-periodic Green's function is not convergent, also known as Rayleigh-Wood frequencies - and of eigenvalues of the Helmholtz transmission problem. Mindless of the several remedies developed to tackle cut-off frequencies through BIEs [14,15,17,19], we choose to avoid them as they are not captured by our previous analysis in [5].

Our discretization method employs a quasi-periodic basis so that techniques forcing the quasi-periodicity of the discrete solutions are not necessary $(c f .[24,46])$. Instead, an accurate approximation of the quasi-periodic Green's function is required in order to extract its Fourier coefficients through the fast Fourier transform (FFT). Moreover, we prove that the chosen discretization basis enjoys a super-algebraic convergence rate on the degrees of freedom, which we then confirm through numerical experiments. In [35], a similar quasi-periodic exponential basis was employed to approximate solutions of a volume integral formulation.

The article is structured as follows. Section 2 presents the notation used throughout as well as the required quasi-periodic Sobolev spaces setting following [5]. In Section 3 we state the Helmholtz transmission problem for a multi-layered grating and study its solvability. Section 4 is concerned with the properties of quasi-periodic boundary integral operators (BIOs) along with an existence and uniqueness result for our BIEs. Section 5 provides rigorous error convergence rates of the spectral method and briefly describes the numerical algorithm used to compute the matrix entries associated with each integral operator. Numerical results are discussed in Section 6 , followed by concluding remarks on Section 7 .

\section{Notation AND FUnCTIONAL SPACE SETting}

\subsection{General notation}

We denote the imaginary unit $\imath$. Boldface symbols signal vectorial quantities while greek and roman letters indicate data over boundaries and volumes, respectively. Canonical vectors in $\mathbb{R}^{2}$ are denoted $\boldsymbol{e}_{\mathbf{1}}, \boldsymbol{e}_{\mathbf{2}}$ respectively. Also, we make use of the symbols $\lesssim, \gtrsim$ and $\cong$ to avoid specifying constants irrelevant for the corresponding analysis.

Let $H$ be a given Banach space. We shall denote its norm as $\|\cdot\|_{H}$ and its dual space by $H^{\prime}$ (set of antilinear functionals over $H$ ) with dual product denoted by $\langle\cdot, \cdot\rangle$. If $H$ is a Hilbert space, the inner product between two of its elements, $x$ and $y$, is written as $(x, y)_{H}$. Moreover, if $H$ is a Hilbert space over the complex field, the inner product will be understood in the anti-linear sense.

For an open domain $\Omega \subset \mathbb{R}^{2}$, its boundary shall be denoted as $\partial \Omega$. Moreover, for any $\mathcal{O} \subset \mathbb{R}^{2}$ such that $\Omega \subseteq \mathcal{O}$, we introduce the closure of $\Omega$ relative to $\mathcal{O}$ as $\bar{\Omega}^{\mathcal{O}}:=\bar{\Omega} \cap \mathcal{O}$ and the boundary of $\Omega$ relative to $\mathcal{O}$ as $\partial^{\mathcal{O}} \bar{\Omega}:=\bar{\Omega}^{\mathcal{O}} \backslash \Omega$.

For $n \in \mathbb{N}_{0}:=\mathbb{N} \cup\{0\}$, we denote by $\mathcal{C}^{n}(\Omega)$ the set of scalar functions over $\Omega$ with complex values and continuous derivatives up to order $n . \mathcal{C}^{\infty}(\Omega)$ refers to the space of functions with infinite continuous derivatives over $\Omega$. We shall also make use of the following subset of $\mathcal{C}^{\infty}(\Omega)$ :

$$
\mathcal{D}(\Omega):=\left\{u \in \mathcal{C}^{\infty}(\Omega): \operatorname{supp} u \subset \subset \Omega\right\} .
$$

The space of $p$-integrable functions (for $p \geq 1$ ) with complex values over $\Omega$ is denoted as $L^{p}(\Omega)$. 
We say that a one-dimensional curve $\Gamma$ is a periodic boundary of class $\mathcal{C}^{r, 1}$, for $r \in \mathbb{N}_{0}$, if it is the restriction of a $x_{1}$-periodic curve $\widetilde{\Gamma}$ that may be parametrized by a periodic function $\boldsymbol{z}: \mathbb{R} \rightarrow \widetilde{\Gamma}$ which has $r$ Lipschitzcontinuous derivatives and a non-vanishing tangential vector. The first derivative of the parametrization is denoted as $\dot{\boldsymbol{z}}$. Moreover, we say that $\Gamma$ is a periodic boundary of class $\mathcal{C}^{\infty}$ if it is of class $C^{r, 1}$ for every $r \in \mathbb{N}_{0}$ - we will also use the notation $\mathcal{C}^{\infty, 1}$ to refer same class, i.e. $\mathcal{C}^{\infty, 1}=\mathcal{C}^{\infty}$.

Throughout, we will consider periodic geometries along $\boldsymbol{e}_{\mathbf{1}}$ with a fixed period of $2 \pi$. Moreover, we say that a continuous function $f$ is a $\theta$-quasi-periodic function if

$$
f\left(\boldsymbol{x}+2 \pi \boldsymbol{e}_{1}\right)=e^{\imath 2 \pi \theta} f(\boldsymbol{x}) \quad \forall \boldsymbol{x} \in \mathbb{R}^{2},
$$

where the quasi-periodic shift $\theta$ is always assumed to be in $[0,1)$. Finally, we define the canonic periodic cell on $\mathbb{R}^{2}$ as $\mathcal{G}:=(0,2 \pi) \times \mathbb{R}$ (see Fig. 1$)$.

\subsection{Quasi-periodic Sobolev spaces}

We denote by $\mathcal{D}_{\theta}\left(\mathbb{R}^{2}\right)$ the space of $\theta$-quasi-periodic functions in $\mathcal{C}^{\infty}\left(\mathbb{R}^{2}\right)$ that vanish for large $\left|x_{2}\right|$, and denote by $\mathcal{D}_{\theta}^{\prime}\left(\mathbb{R}^{2}\right)$ the space of $\theta$-quasi-periodic distributions, which can be seen as the dual space of $\mathcal{D}_{\theta}\left(\mathbb{R}^{2}\right)(c f$. [5], Prop. 2.4). For $\mathcal{G}$ as before, we introduce $\mathcal{D}_{\theta}(\mathcal{G})$ the space of restrictions to $\mathcal{G}$ of elements in $\mathcal{D}_{\theta}\left(\mathbb{R}^{2}\right)$. Moreover, for any open domain $\Omega \subset \mathcal{G}$ we define $\mathcal{D}_{\theta}(\Omega)$ as the set of elements of $\mathcal{D}_{\theta}(\mathcal{G})$ with compact support on $\Omega$ and $\mathcal{D}_{\theta}^{\prime}(\Omega)$ as the space of elements of $\mathcal{D}_{\theta}^{\prime}(\mathcal{G})$ restricted to $\mathcal{D}_{\theta}(\Omega)$. In what follows, for all $j \in \mathbb{Z}$ we define $j_{\theta}:=j+\theta$.

Proposition 2.1 ([5, Prop. 2.6]). Every $u \in \mathcal{D}_{\theta}\left(\mathbb{R}^{2}\right)$ can be represented as a Fourier series, i.e.,

$$
u(\boldsymbol{x})=\sum_{j \in \mathbb{Z}} u_{j}\left(x_{2}\right) e^{i j_{\theta} x_{1}} \quad \text { with } \quad u_{j}\left(x_{2}\right):=\frac{1}{2 \pi} \int_{0}^{2 \pi} e^{-\imath j_{\theta} x_{1}} u(\boldsymbol{x}) \mathrm{d} x_{1},
$$

so that $u_{j} \in \mathcal{D}(\mathbb{R})$. On the other hand, every element $F \in \mathcal{D}_{\theta}^{\prime}\left(\mathbb{R}^{2}\right)$ can be identified with a formal Fourier series given by

$$
\sum_{j \in \mathbb{Z}} F_{j} e^{i j_{\theta} x_{1}} \quad \text { with } \quad F_{j}:= \begin{cases}\mathcal{D}(\mathbb{R}) & \rightarrow \mathbb{C} \\ v & \mapsto F\left(v\left(x_{2}\right) e^{i j_{\theta} x_{1}}\right)\end{cases}
$$

where $F_{j} \in \mathcal{D}^{\prime}(\mathbb{R})$ for all $j \in \mathbb{Z}$ and $F(u)=\sum_{j \in \mathbb{Z}} F_{j}\left(u_{j}\right)$.

Let $s \in \mathbb{R}$. We define the $\theta$-quasi-periodic Sobolev space of order $s$ on $\mathcal{G}$ as follows,

$$
H_{\theta}^{s}(\mathcal{G}):=\left\{\left.F \in \mathcal{D}_{\theta}^{\prime}\left(\mathbb{R}^{2}\right)\left|\sum_{j \in \mathbb{Z}} \int_{\mathbb{R}}\left(1+j_{\theta}^{2}+|\xi|^{2}\right)^{s}\right| \widehat{F}_{j}(\xi)\right|^{2} \mathrm{~d} \xi<\infty\right\},
$$

wherein $\widehat{F}_{j}$ is the Fourier transform (in distributional sense [42], Sect. 2.4) of $F_{j}$, defined as in Proposition 2.1. Additionally, we introduce the common notation $L_{\theta}^{2}(\mathcal{G}):=H_{\theta}^{0}(\mathcal{G})$ and note that, as in the standard case, $H_{\theta}^{s}(\mathcal{G})$ is a Hilbert space ([5], Prop. 2.8). Furthermore, for an open proper subset $\Omega$ of $\mathcal{G}$, we define $H_{\theta}^{s}(\Omega)$ as the Hilbert space of restrictions to $\Omega$ of elements of $H_{\theta}^{s}(\mathcal{G})$ (see [5], Sect. 2 and [32], Chap. 3.6). Finally, local Sobolev spaces on $\Omega$ are defined as

$$
H_{\theta, \mathrm{loc}}^{s}(\Omega):=\left\{u \in \mathcal{D}_{\theta}^{\prime}(\Omega): u \in H_{\theta}^{s}\left(\Omega \cap\left\{\boldsymbol{x} \in \mathcal{G}:\left|x_{2}\right|<R\right\}\right) \quad \forall R>0\right\} .
$$




\subsection{Quasi-periodic Sobolev spaces on boundaries and traces}

We begin by considering spaces of periodic functions over $\mathbb{R}$. As in Definition 8.1 of [28], Section 5.3 of [37], we define Sobolev spaces on $[0,2 \pi]$ of order $s \geq 0$ as follows,

$$
H^{s}[0,2 \pi]:=\left\{\phi \in L^{2}((0,2 \pi)): \sum_{j \in \mathbb{Z}}\left(1+j^{2}\right)^{s}\left|\phi_{j}\right|^{2}<\infty\right\},
$$

where $\left\{\phi_{j}\right\}_{j \in \mathbb{Z}}$ are the Fourier coefficients of $\phi$. Quasi-periodic spaces of order $s \geq 0$ over $(0,2 \pi)$ are defined from $H^{s}[0,2 \pi]$ straightforwardly, i.e.,

$$
H_{\theta}^{s}[0,2 \pi]:=\left\{\phi \in L^{2}((0,2 \pi)): e^{-\imath \theta t} \phi(t) \in H^{s}[0,2 \pi]\right\} .
$$

Both $H^{s}[0,2 \pi]$ and $H_{\theta}^{s}[0,2 \pi]$ are Hilbert spaces, as are their respective dual spaces, denoted respectively $H^{-s}[0,2 \pi]$ and $H_{\theta}^{-s}[0,2 \pi]$ (see [28], Thm. 8.10 and [5], Thm. 2.20). Moreover, for $s \in \mathbb{R}$, the inner product and norm of $H_{\theta}^{s}[0,2 \pi]$ are given by:

$$
(u, v)_{H_{\theta}^{s}[0,2 \pi]}:=\sum_{j \in \mathbb{Z}}\left(1+j_{\theta}^{2}\right)^{s} u_{j, \theta} \overline{v_{j, \theta}} \quad \text { and } \quad\|u\|_{H_{\theta}^{s}[0,2 \pi]}:=(u, u)_{H_{\theta}^{s}[0,2 \pi]}^{\frac{1}{2}},
$$

wherein, for positive $s$, we define

$$
u_{j, \theta}:=\frac{1}{2 \pi}\left(u(t), e^{\imath j_{\theta} t}\right)_{L^{2}((0,2 \pi))},
$$

and the product is extended through duality to negative $s$ (cf. [5], Thms. 2.16 and 2.20).

Let $\Gamma$ be a periodic boundary of class $\mathcal{C}^{\infty}$ and let $z:(0,2 \pi) \rightarrow \Gamma$ be a $\mathcal{C}^{\infty}$ parametrization of $\Gamma$. Then, for any $s \geq 0$, we define the $\theta$-quasi-periodic Sobolev space of order $s$ on $\Gamma$ as

$$
H_{\theta}^{s}(\Gamma):=\left\{u \in L_{\theta}^{2}(\Gamma) \mid(u \circ \boldsymbol{z})(t) \in H_{\theta}^{s}[0,2 \pi]\right\},
$$

with norm,

$$
\|u\|_{H_{\theta}^{s}(\Gamma)}:=\|u \circ \boldsymbol{z}\|_{H_{\theta}^{s}[0,2 \pi]} .
$$

We define $H_{\theta}^{-s}(\Gamma)$ as the completion of $L_{\theta}^{2}(\Gamma)$ under the norm given by

$$
\|u\|_{H_{\theta}^{-s}(\Gamma)}:=\|(u \circ \boldsymbol{z})\| \dot{\boldsymbol{z}}\left\|_{\mathbb{R}^{2}}\right\|_{H_{\theta}^{-s}[0,2 \pi]} .
$$

Norms and inner products for these spaces are given through their respective pullbacks to $H_{\theta}^{s}[0,2 \pi]$ and $H_{\theta}^{-s}[0,2 \pi]$. Moreover, $H_{\theta}^{-s}(\Gamma)$ is identified with the dual space of $H_{\theta}^{s}(\Gamma)$ ([5], Thm. 2.26) where the duality is given by the extension of the following anti-linear form:

$$
\langle\lambda, \vartheta\rangle_{\Gamma}:=(\lambda, \vartheta)_{L_{\theta}^{2}(\Gamma)}, \quad \lambda, \vartheta \in L_{\theta}^{2}(\Gamma)
$$

We also define the following space of smooth functions over $\Gamma$,

$$
\mathcal{D}_{\theta}(\Gamma):=\left\{\phi: \Gamma \rightarrow \mathbb{C} \mid(\phi \circ \boldsymbol{z})(t)=\sum_{j=-n}^{n} \phi_{j} e^{i j_{\theta} t}, \text { for some } n \in \mathbb{N}\right\},
$$

which is dense in $H_{\theta}^{s}(\Gamma)$ for any $s \in \mathbb{R}$. 


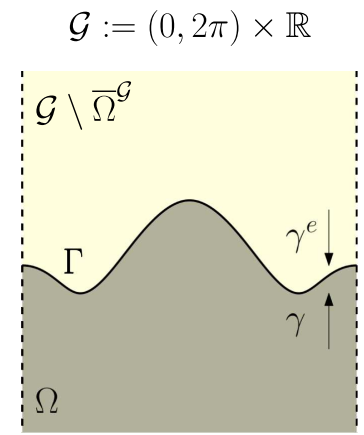

FigURE 1. Example of a domain $\Omega \subset \mathcal{G}$ with $\partial^{\mathcal{G}} \Omega=\Gamma$. $\mathcal{G}$ encompasses the unbounded area between dashed lines, while $\Omega$ and $\mathcal{G} \backslash \bar{\Omega}^{\mathcal{G}}$ correspond to the shaded and unshaded portions, respectively. Trace operators $\gamma$ and $\gamma^{e}$ act from $\Omega$ and $\mathcal{G} \backslash \bar{\Omega}^{\mathcal{G}}$ towards $\Gamma$, respectively.

Finally, we introduce trace operators acting on quasi-periodic Sobolev spaces. Let $\Omega$ be a proper, open subset of $\mathcal{G}$ such that $\partial^{\mathcal{G}} \Omega=\Gamma$, we define the following operators for $s>\frac{1}{2}$ :

$$
\gamma_{\mathrm{D}}: H_{\theta}^{s}(\Omega) \rightarrow H_{\theta}^{s-\frac{1}{2}}(\Gamma), \quad \gamma_{\mathrm{D}}^{e}: H_{\theta}^{s}\left(\mathcal{G} \backslash \bar{\Omega}^{\mathcal{G}}\right) \rightarrow H_{\theta}^{s-\frac{1}{2}}(\Gamma),
$$

that extend the notion of the restriction operator $\left.u \mapsto u\right|_{\Gamma}$ to quasi-periodic Sobolev spaces ([5], Thm. 2.29). In this context, $\gamma_{\mathrm{D}}$ and $\gamma_{\mathrm{D}}^{e}$ are, respectively, the interior and exterior Dirichlet traces. Analogously, for $s>\frac{3}{2}$, we denote the interior and exterior Neumann traces on $\Omega$ as

$$
\gamma_{\mathrm{N}}: H_{\theta}^{s}(\Omega) \rightarrow H_{\theta}^{s-\frac{3}{2}}(\Gamma), \quad \gamma_{\mathrm{N}}^{e}: H_{\theta}^{s}\left(\mathcal{G} \backslash \bar{\Omega}^{\mathcal{G}}\right) \rightarrow H_{\theta}^{s-\frac{3}{2}}(\Gamma),
$$

extending the normal derivative $\left.u \mapsto \nabla u\right|_{\Gamma} \cdot \boldsymbol{n}$, where $\boldsymbol{n}$ is - for both traces - the unitary normal exterior to $\Omega$. Moreover, introducing the subspace of elements of $H_{\theta}^{1}(\Omega)$ with integrable Laplacian,

$$
H_{\theta, \Delta}^{s}(\Omega):=\left\{u \in H_{\theta}^{1}(\Omega): \Delta u \in L_{\theta}^{2}(\Omega)\right\},
$$

the Neumann trace may be extended as

$$
\gamma_{\mathrm{N}}: H_{\theta, \Delta}^{1}(\Omega) \rightarrow H_{\theta}^{-\frac{1}{2}}(\Gamma), \quad \gamma_{\mathrm{N}}^{e}: H_{\theta, \Delta}^{1}\left(\mathcal{G} \backslash \bar{\Omega}^{\mathcal{G}}\right) \rightarrow H_{\theta}^{-\frac{1}{2}}(\Gamma),
$$

through integration by parts (cf. [5], Sect. 2). All the previous results concerning trace operators follow analogously (with obvious modifications) for both local spaces - in the case that $\Omega$ is unbounded - and if $\Omega$ is the bounded space between two non-intersecting periodic curves $\Gamma_{1}$ and $\Gamma_{2}$. Finally, we denote the following vector operators

$$
\gamma u:=\left(\gamma_{\mathrm{D}} u, \gamma_{\mathrm{N}} u\right)^{t}, \quad \gamma^{e} u:=\left(\gamma_{\mathrm{D}}^{e} u, \gamma_{\mathrm{N}}^{e} u\right)^{t} \quad \text { and } \quad[\gamma u]_{\Gamma}:=\gamma^{e} u-\gamma u,
$$

as interior, exterior and jump trace vectors on $\Gamma$, respectively. Figure 1 exemplifies the aforementioned setting as well as the action of the trace operators.

\section{Helmholtz PRoblem in PeRiodic LAYEREd Media}

\subsection{Geometric setting}

We seek to establish a boundary integral representation for scattered and transmitted acoustic or electromagnetic fields resulting from plane waves impinging a multi-layered grating. The domain is described by $M \in \mathbb{N}$ 


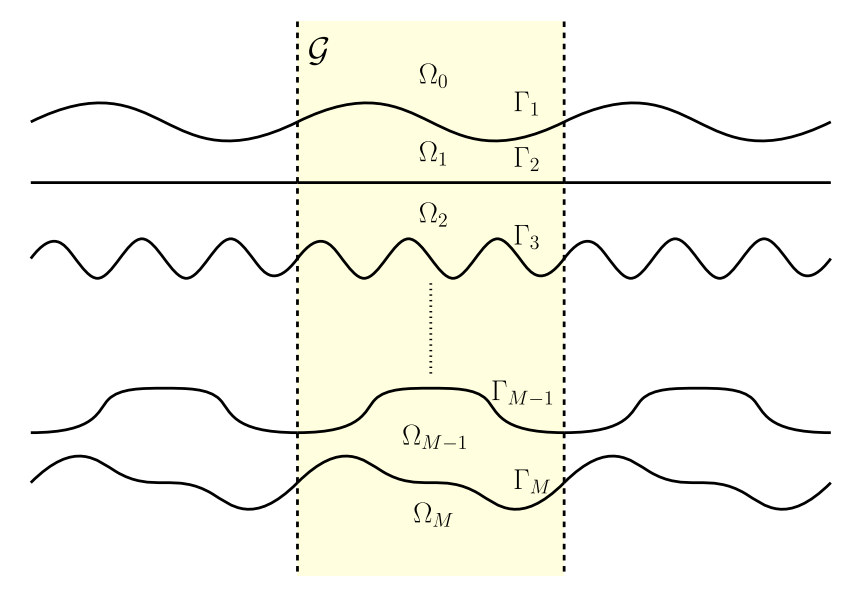

FIgURE 2. Example of a multi-layered grating. $\mathcal{G}$ is highlighted and the dashed lines represent its boundaries at 0 and $2 \pi$.

finite non-intersecting periodic surfaces $\left\{\widetilde{\Gamma}_{i}\right\}_{i=1}^{M}$ - ordered downwards - separating $M+1$ periodic domains $\left\{\widetilde{\Omega}_{i}\right\}_{i=0}^{M}$ such that for $0<i<M$ it holds $\partial \widetilde{\Omega}_{i}=\widetilde{\Gamma}_{i} \cup \widetilde{\Gamma}_{i+1}, \partial \widetilde{\Omega}_{0}=\widetilde{\Gamma}_{1}$ and $\partial \widetilde{\Omega}_{M}=\widetilde{\Gamma}_{M}$ (see Fig. 2). Moreover, while all domains $\left\{\widetilde{\Omega}_{i}\right\}_{i=0}^{M}$ are unbounded along $\boldsymbol{e}_{1}-$ due to their periodicity - only two of them, namely $\widetilde{\Omega}_{0}$ and $\widetilde{\Omega}_{M}$, are unbounded in the second spatial dimension (along $\boldsymbol{e}_{2}$ ). The restrictions of the aforementioned domains and surfaces to the periodic cell $\mathcal{G}$ are denoted by:

$$
\Omega_{i}:=\widetilde{\Omega}_{i} \cap \mathcal{G} \quad \forall i \in\{0, \ldots, M\}, \quad \Gamma_{j}:=\widetilde{\Gamma}_{j} \cap \mathcal{G} \quad \forall j \in\{1, \ldots, M\} .
$$

Additionally, we choose $H>0$ so that

$$
\bigcup_{i=1}^{M-1} \bar{\Omega}_{i}^{\mathcal{G}} \subset\left\{\boldsymbol{x} \in \mathcal{G}:\left|x_{2}\right|<H\right\}
$$

holds. We will assume that the interfaces $\Gamma_{i}, i \in\{1, \ldots, M\}$, are all periodic boundaries of class $\mathcal{C}^{\infty}$. Furthermore, for each $i \in\{1, \ldots, M\}$, the exterior and interior trace operators on $\Gamma_{i}$ are understood as

$$
\begin{gathered}
\gamma_{\mathrm{D}}^{e}: H_{\theta}^{1}\left(\Omega_{i-1}\right) \rightarrow H_{\theta}^{\frac{1}{2}}\left(\Gamma_{i}\right), \quad \gamma_{\mathrm{D}}: H_{\theta}^{1}\left(\Omega_{i}\right) \rightarrow H_{\theta}^{\frac{1}{2}}\left(\Gamma_{i}\right), \\
\gamma_{\mathrm{N}}^{e}: H_{\theta, \Delta}^{1}\left(\Omega_{i-1}\right) \rightarrow H_{\theta}^{-\frac{1}{2}}\left(\Gamma_{i}\right) \quad \text { and } \quad \gamma_{\mathrm{N}}: H_{\theta, \Delta}^{1}\left(\Omega_{i}\right) \rightarrow H_{\theta}^{-\frac{1}{2}}\left(\Gamma_{i}\right),
\end{gathered}
$$

and the normal vector on $\Gamma_{i}$ is chosen to point towards $\Omega_{i-1}$.

\subsection{Helmholtz transmission problem on periodic media}

For a time-dependence $e^{-\imath \omega t}$ for some frequency $\omega>0$, let the previously described grating be illuminated by an incident plane wave,

$$
u^{(\mathrm{inc})}(\boldsymbol{x}):=e^{\imath \mathbf{k}_{\mathbf{0}} \cdot \boldsymbol{x}}=e^{\imath\left(k_{0,1} x_{1}+k_{0,2} x_{2}\right)},
$$

where $\mathbf{k}_{0}=\left(k_{0,1}, k_{0,2}\right) \in \mathbb{R}^{2}$. Furthermore, we denote $k_{0}:=\left|\mathbf{k}_{\mathbf{0}}\right|$.

For $i=0, \ldots, M$, the material filling each domain $\Omega_{i}$ is assumed to be homogeneous and isotropic with refraction index $\eta_{i}$ - we assume $\eta_{0} \equiv 1-$ and wavenumber $k_{i}:=\omega c_{i}^{-1}=\eta_{i} k_{0}$, where $c_{i}$ is the wave speed in $\Omega_{i}$. 
Throughout this section, we fix $\theta$ as the unique real in $[0,1)$ such that $\theta=k_{0,1}+n$ for some integer $n$ and, for all $j \in \mathbb{Z}$, we define

$$
\beta_{j}^{(0)}:=\left\{\begin{array}{ll}
\sqrt{k_{0}^{2}-j_{\theta}^{2}} & \text { if } k_{0}^{2}-j_{\theta}^{2} \geq 0 \\
\imath \sqrt{j_{\theta}^{2}-k_{0}^{2}} & \text { if } k_{0}^{2}-j_{\theta}^{2}<0
\end{array}, \quad \beta_{j}^{(M)}:=\left\{\begin{array}{ll}
\sqrt{k_{M}^{2}-j_{\theta}^{2}} & \text { if } k_{M}^{2}-j_{\theta}^{2} \geq 0 \\
\imath \sqrt{j_{\theta}^{2}-k_{M}^{2}} & \text { if } k_{M}^{2}-j_{\theta}^{2}<0
\end{array},\right.\right.
$$

where as before $j_{\theta}=j+\theta$. With these definitions, we can state our volume problem as follows.

Problem 3.1 (Helmholtz transmission problem). We seek $u^{(\text {tot })}$ defined as

$$
u^{(\mathrm{tot})}:= \begin{cases}u^{(\mathrm{inc})}+u_{0} & \text { in } \Omega_{0}, \\ u_{i} & \text { in } \Omega_{i} \text { for } i \in\{1, \ldots, M\},\end{cases}
$$

where $u_{0} \in H_{\theta, \text { loc }}^{1}\left(\Omega_{0}\right), u_{M} \in H_{\theta, \text { loc }}^{1}\left(\Omega_{M}\right)$ and $u_{i} \in H_{\theta}^{1}\left(\Omega_{i}\right)$ for all $1 \leq i \leq M-1$, such that

$$
\begin{aligned}
-\left(\Delta+k_{i}^{2}\right) u^{(\text {tot })} & =0 \quad \text { in } \Omega_{i} \cap\left\{\boldsymbol{x} \in \mathcal{G}:\left|x_{2}\right| \leq H\right\}, \quad \forall i \in\{0, \ldots, M\}, \\
{\left[\gamma u^{(\text {tot })}\right]_{\Gamma_{i}} } & =0 \quad \text { on } \Gamma_{i}, \quad \forall i \in\{1, \ldots, M\}, \\
u_{0}(\boldsymbol{x}) & =\sum_{j \in \mathbb{Z}} u_{j}^{(0)} e^{\imath\left(\beta_{j}^{(0)}\left(x_{2}-H\right)+j_{\theta} x_{1}\right)} \quad \text { for } x_{2} \geq H \\
u_{M}(\boldsymbol{x}) & =\sum_{j \in \mathbb{Z}} u_{j}^{(M)} e^{\imath\left(-\beta_{j}^{(M)}\left(x_{2}+H\right)+j_{\theta} x_{1}\right)} \quad \text { for } x_{2} \leq-H .
\end{aligned}
$$

Equation (3.2b) represents the continuity of Dirichlet and Neumann traces across each interface. This condition can be generalized to include different transmission coefficients without much effort. The last two conditions, namely (3.2c) and (3.2d), correspond to radiation conditions for $u_{0}$ and $u_{M}$, also known as the Rayleigh-Bloch expansions (cf. [34] for a detailed discussion), where $\left\{u_{j}^{(0)}\right\}_{j \in \mathbb{Z}}$ and $\left\{u_{j}^{(M)}\right\}_{j \in \mathbb{Z}}$ are the corresponding Rayleigh coefficients.

Through an analogous analysis to that presented in ([22], Sect. 3), one finds that - for a fixed choice of geometries $\left\{\Gamma_{i}\right\}_{i=1}^{M}$ and refraction indices $\left\{\eta_{i}\right\}_{i=1}^{M}$ - Problem 3.1 has a unique solution for all but a countable number of wavenumbers $k_{0}$ as all wavenumbers $k_{i}$ for $i \in\{1, \ldots, M\}$ depend on $k_{0}$.

Assumption 3.2. The wavenumber $k_{0}$ is such that Problem 3.1 has a unique solution.

We shall make no further analysis of the volume problem as stated above, and limit ourselves to [7,10,22,27, $34,41,45]$ and references therein for more detailed analyses of the radiation condition of similar problems.

Remark 3.3. The restriction of the Helmholtz transmission problem in an infinite $x_{1}$-periodic domain to a single period is made possible by the quasi-periodicity of the plane wave $u^{(\mathrm{inc})}$. However, the same restriction, as well as the forthcoming analysis, holds for any quasi-periodic boundary data.

\section{BOUNDARY INTEGRAL EQUATIONS}

Following our previous work [5], we introduce the quasi-periodic Green's function and recall some relevant properties. We then define the quasi-periodic single and double layer potentials and boundary integral operators (BIOs) arising from taking their respective traces on the periodic boundaries $\left\{\Gamma_{i}\right\}_{i=1}^{M}$. To conclude this section, we present an integral representation for the fields $\left\{u_{i}\right\}_{i=0}^{M}$ and a proof of unisolvency for the corresponding BIE. As before, $\theta \in[0,1)$ will denote the quasi-periodic shift. 


\subsection{Quasi-periodic fundamental solution}

Consider a positive wavenumber $k \in \mathbb{R}$, we recall the definition of the cut-off frequencies.

Definition 4.1. We say $k>0$ is a cut-off frequency, if there is $j \in \mathbb{Z}$, such that

$$
|j+\theta|=k,
$$

where $\theta$ is the previously fixed quasi-periodic shift.

These frequencies correspond to values where the quasi-periodic Green's function can not be represented in a traditional manner. While a number of alternatives have been developed to circumvent this issue, e.g., $[15,17,19]$, their analysis is out of the scope of our present work. Hence, in what follows, we make the next assumption.

Assumption 4.2. The wavenumber $k>0$ is not a cut-off frequency for the given $\theta \in[0,1)$.

Under Assumption 4.2 we can define the $\theta$-quasi-periodic Green's function as (cf. $[29,34]$ and references therein)

$$
G_{\theta}^{k}(\boldsymbol{x}, \boldsymbol{y}):=\lim _{m \rightarrow \infty} \sum_{n=-m}^{m} e^{-\imath 2 \pi n \theta} G^{k}\left(\boldsymbol{x}+2 \pi n \boldsymbol{e}_{1}, \boldsymbol{y}\right),
$$

for all $\boldsymbol{x}, \boldsymbol{y}$ in $\mathbb{R}^{2}$ such that $\boldsymbol{x}-\boldsymbol{y} \neq 2 \pi n \boldsymbol{e}_{1}$ for all $n \in \mathbb{Z}$, wherein $G^{k}(\boldsymbol{x}, \boldsymbol{y})$ is the fundamental solution for the Helmholtz equation with wavenumber $k$, namely,

$$
G^{k}(\boldsymbol{x}, \boldsymbol{y})=\frac{\imath}{4} H_{0}^{(1)}\left(k\|\boldsymbol{x}-\boldsymbol{y}\|_{\mathbb{R}^{2}}\right)
$$

where $H_{0}^{(1)}(\cdot)$ denotes the zeroth-order first kind Hankel function. Moreover, the quasi-periodic Green's function is a fundamental solution of the Helmholtz equation in the following sense:

$$
-\left(\Delta_{\boldsymbol{y}}+k^{2}\right) G_{\theta}^{k}(\boldsymbol{x}, \boldsymbol{y})=\sum_{n \in \mathbb{Z}} \delta\left(\boldsymbol{x}+2 \pi n \boldsymbol{e}_{1}\right) e^{22 \pi n \theta}
$$

for all $\boldsymbol{x} \in \mathbb{R}^{2}$, satisfying the radiation condition specified in the preceding section (cf. [34], Prop. 3.1).

Remark 4.3. If Assumption 4.2 is not met, the sum in (4.2) fails to converge for any pair of $\boldsymbol{x}, \boldsymbol{y} \in \mathbb{R}^{2}$.

\subsection{Layer potentials and boundary integral operators}

In this section, we will assume a given boundary $\Gamma$ satisfying the following assumption.

Assumption 4.4. Given $r \in[0, \infty]$, the interface $\Gamma$ is a periodic boundary of class $\mathcal{C}^{r, 1}$.

We denote by $\Omega$ the part of $\mathcal{G}$ below $\Gamma$ (see Fig. 2). For $\phi \in \mathcal{D}_{\theta}(\Gamma)$, one can define the single and double layer potentials as

$$
\mathrm{SL}_{\theta, \Gamma}^{k} \phi(\boldsymbol{x}):=\int_{\Gamma} G_{\theta}^{k}(\boldsymbol{x}, \boldsymbol{y}) \phi(\boldsymbol{y}) \mathrm{d} \boldsymbol{y}, \quad \mathrm{DL}_{\theta, \Gamma}^{k} \phi(\boldsymbol{x}):=\int_{\Gamma} \gamma_{\mathrm{N}, \boldsymbol{y}} G_{\theta}^{k}(\boldsymbol{x}, \boldsymbol{y}) \phi(\boldsymbol{y}) \mathrm{d} \boldsymbol{y}
$$

where $\gamma_{n, \boldsymbol{y}}$ denotes the interior (with respect to $\Omega$ ) Neumann trace operator acting on functions with argument $\boldsymbol{y}$.

Lemma 4.5 ([5], Thms. 4.7 and 4.10). Let $k$ and $\Gamma$ satisfy Assumptions 4.2 and 4.4 with $r \geq 0$, respectively. Then, the single and double layer potentials can be extended as continuous operators acting on Sobolev spaces as follows

$$
\mathrm{SL}_{\theta, \Gamma}^{k}: H_{\theta}^{s-\frac{1}{2}}(\Gamma) \rightarrow H_{\theta, l o c}^{s+1}(\mathcal{G}) \quad \text { and } \quad \mathrm{DL}_{\theta, \Gamma}^{k}: H_{\theta}^{s+\frac{1}{2}}(\Gamma) \rightarrow H_{\theta, l o c}^{s+1}(\mathcal{G} \backslash \Gamma), \quad \text { for } s<\frac{1}{2}
$$


We then define BIOs by taking traces of the layer potentials:

$$
\begin{aligned}
\mathrm{V}_{\theta, \Gamma}^{k}:=\gamma_{\mathrm{D}} \mathrm{SL}_{\theta, \Gamma} & \mathrm{K}_{\theta, \Gamma}^{\prime k}:=\gamma_{\mathrm{N}} \mathrm{SL}_{\theta, \Gamma}+\frac{1}{2} \mathrm{I} \\
\mathrm{W}_{\theta, \Gamma}^{k}:=-\gamma_{\mathrm{N}} \mathrm{DL}_{\theta, \Gamma} & \mathrm{K}_{\theta, \Gamma}^{k}:=\gamma_{\mathrm{D}} \mathrm{DL}_{\theta, \Gamma}-\frac{1}{2} \mathrm{I}
\end{aligned}
$$

Moreover, due to the jump properties of the layer potentials ([5], Lem. 4.11), the following relations hold:

$$
\begin{aligned}
\mathrm{V}_{\theta, \Gamma}^{k}=\gamma_{\mathrm{D}}^{e} \mathrm{SL}_{\theta, \Gamma}, & \mathrm{K}_{\theta, \Gamma}^{\prime k}=\gamma_{\mathrm{N}}^{e} \mathrm{SL}_{\theta, \Gamma}-\frac{1}{2} \mathrm{I}, \\
\mathrm{W}_{\theta, \Gamma}^{k}=-\gamma_{\mathrm{N}}^{e} \mathrm{DL}_{\theta, \Gamma}, & \mathrm{K}_{\theta, \Gamma}^{k}=\gamma_{\mathrm{D}}^{e} \mathrm{DL}_{\theta, \Gamma}+\frac{1}{2} \mathrm{I} .
\end{aligned}
$$

Remark 4.6. When considering interior and exterior traces acting on layer potentials, note that the normal vector on $\Gamma$ is fixed so that the only difference between exterior and interior traces is the direction from which we approach $\Gamma$. Additionally, note that, having fixed the normal vector to $\Gamma$, the choice of trace taken in the definition of $\mathrm{W}_{\theta, \Gamma}^{k}$ is arbitrary and makes no difference.

Lemma 4.7 ([5], Thm. 4.10). Let $k$ and $\Gamma$ be as in Assumptions 4.2 and 4.4 with $r \geq 0$, respectively. Then, for $|s|<\frac{1}{2}$, the BIOs satisfy the following continuity conditions

$$
\begin{gathered}
\mathrm{V}_{\theta, \Gamma}^{k}: H_{\theta}^{s-\frac{1}{2}}(\Gamma) \rightarrow H_{\theta}^{s+\frac{1}{2}}(\Gamma), \quad \mathrm{W}_{\theta, \Gamma}^{k}: H_{\theta}^{s+\frac{1}{2}}(\Gamma) \rightarrow H_{\theta}^{s-\frac{1}{2}}(\Gamma), \\
\mathrm{K}_{\theta, \Gamma}^{\prime k}: H_{\theta}^{s-\frac{1}{2}}(\Gamma) \rightarrow H_{\theta}^{s-\frac{1}{2}}(\Gamma), \quad \mathrm{K}_{\theta, \Gamma}^{k}: H_{\theta}^{s+\frac{1}{2}}(\Gamma) \rightarrow H_{\theta}^{s+\frac{1}{2}}(\Gamma) .
\end{gathered}
$$

\subsubsection{Compacteness Properties}

Until this point, we have established continuity properties of the four BIOs defined in (4.4). However, the BIEs we consider in the coming section require the subtraction of two instances of the same BIO with different wavenumbers. This requires a number of results from pseudo-differential operator theory [37] as well as a version of the Rellich theorem on quasi-periodic Sobolev spaces on boundaries. We show that the difference between any two of the operators in (4.4) - with different wavenumbers - will result in a compact operator.

Theorem 4.8 (Rellich Theorem for quasi-periodic Sobolev spaces). Let $s_{1}, s_{2}$ be real numbers such that $s_{1}<s_{2}$ and $\theta \in[0,1)$. Then, $H_{\theta}^{s_{2}}(\Gamma)$ is compactly embedded in $H_{\theta}^{s_{1}}(\Gamma)$.

Proof. Follows directly from the definition of the quasi-periodic spaces and the result for standard Sobolev spaces (see [28], Thm. 8.3).

Remark 4.9. No smoothness assumptions are needed for the proof of the previous theorem. Thus, it can be extended to Lipschitz boundaries for any pair of real numbers $s_{1}, s_{2}<1$, and potentially less regular cases if we restrict $s_{1}, s_{2}$ to be non-negative.

Theorem 4.10 ([37], Thm. 6.1.1). Let $a: \mathbb{R} \times \mathbb{R} \rightarrow \mathbb{C}$ be a $2 \pi$-bi-periodic function of class $\mathcal{C}^{\infty}$ and $S$ be a $2 \pi$-periodic distribution in $\mathbb{R}$. Consider the following formal operator acting on a $2 \pi$-periodic smooth function $u \in \mathcal{C}^{\infty}(\mathbb{R}):$

$$
A u(s)=\int_{0}^{2 \pi} S(s-t) a(s, t) u(t) d t \quad \forall s \in \mathbb{R},
$$

where integration is to be understood as a duality pairing. Furthermore, let us assume the Fourier coefficients of $S$ to behave as

$$
\left|S_{n}\right| \lesssim|n|^{p},
$$


for some $p \in \mathbb{R}$. Then, for any $s \in \mathbb{R}, A$ in (4.6) may be continuously extended as an operator mapping from $H^{s}[0,2 \pi]$ to $H^{s-p}[0,2 \pi]$, i.e.,

$$
A: H^{s}[0,2 \pi] \rightarrow H^{s-p}[0,2 \pi] .
$$

We also recall a classical result from Fourier analysis ( $c f$. [43]).

Lemma 4.11. Let $m \in \mathbb{N}, f: \mathbb{R} \rightarrow \mathbb{C}$ be a periodic $\mathcal{C}^{m}$-class function such that its distributional derivative of order $m+1$ belongs to $L^{1}((0,2 \pi))$. Then, its Fourier coefficients $\left\{f_{n}\right\}_{n \in \mathbb{Z}}$ are such that

$$
\left|f_{n}\right| \lesssim|n|^{-m-1}
$$

In order to employ Theorem 4.10 we will need to express the quasi-periodic BIOs in a convenient way: with periodic functions as kernels. Let $k$ and $\Gamma$ be as in Assumptions 4.2 and 4.4, respectively. We begin by considering a periodic version of the fundamental solution in (4.2) and its derivatives on $\Gamma$ as

$$
\widehat{G}_{\theta}^{k}(s, t):=e^{-\imath \theta(s-t)} G_{\theta}^{k}(\boldsymbol{z}(s), \boldsymbol{z}(t)),
$$

which may be expressed as

$$
\widehat{G}_{\theta}^{k}(s, t)=S(t-s) J_{\theta}^{k}(s, t)+R_{\theta}^{k}(s, t)
$$

with

$$
\begin{aligned}
S(t) & :=-\frac{1}{2 \pi} \log \left|2 \sin \frac{|t|}{2}\right| \\
J_{\theta}^{k}(s, t) & :=e^{-\imath \theta(s-t)} \sum_{j=-\infty}^{\infty} J_{0}\left(k\left\|\boldsymbol{z}(s)+2 \pi j \boldsymbol{e}_{\mathbf{1}}-\boldsymbol{z}(t)\right\|\right) e^{-\imath 2 \pi j \theta} \chi_{\epsilon}(s-t+2 \pi j),
\end{aligned}
$$

where $J_{0}(\cdot)$ is the zeroth-first kind Bessel function, $\epsilon \in(0,2 \pi)$ and $\chi_{\epsilon}(\cdot)$ is a smooth function satisfying

$$
\chi_{\epsilon}(s)=0 \quad \text { if } \quad|s|>\epsilon \quad \text { and } \quad \chi_{\epsilon}(s)=1 \quad \text { if } \quad|s|<\frac{1}{2} \epsilon
$$

and

$$
R_{\theta}^{k}(s, t)=\widehat{G}_{\theta}^{k}(s, t)-S(t-s) J_{\theta}^{k}(s, t) .
$$

Using known expansions of Hankel functions (see [1], 9.1.12-9.1.13) one can check that $R_{\theta}^{k}$ belongs to $\mathcal{C}^{\infty}(\mathbb{R} \times \mathbb{R})$.

Before we proceed any further, it is necessary to introduce a second wavenumber. We will denote $\widetilde{k}>0$ a wavenumber (not necessarily different from $k$ ) that also satisfies Assumption 4.2.

Proposition 4.12. Let $k$ and $\widetilde{k}$ satisfy Assumption 4.2, and let $\Gamma$ satisfy Assumption 4.4 with $r=\infty$. Consider $\mathrm{V}_{\theta}^{k}$ and $\mathrm{V}_{\theta}^{\widetilde{k}}$ the weakly singular BIOs on $\Gamma$ defined in (4.4) and where we have dropped the $\Gamma$ subscript for brevity. Both operators may be considered as pseudo-differential operators of order -1, whence

$$
\mathrm{V}_{\theta}^{k}: H_{\theta}^{s}(\Gamma) \rightarrow H_{\theta}^{s+1}(\Gamma), \quad \mathrm{V}_{\theta}^{\widetilde{k}}: H_{\theta}^{s}(\Gamma) \rightarrow H_{\theta}^{s+1}(\Gamma) .
$$

Moreover, the operator $\mathrm{V}_{\theta}^{k, \widetilde{k}}:=\mathrm{V}_{\theta}^{k}-\mathrm{V}_{\theta}^{\widetilde{k}}$ can be extended to

$$
\mathrm{V}_{\theta}^{k, \widetilde{k}}: H_{\theta}^{s}(\Gamma) \rightarrow H_{\theta}^{s+3}(\Gamma),
$$

as a bounded linear operator for every $s \in \mathbb{R}$. 
Proof. That $\mathrm{V}_{\theta}^{k}$ (and $\mathrm{V}_{\theta}^{\widetilde{k}}$ ) may be extended as claimed follows directly from Theorem 4.10, the kernel representation (4.8) and the decay of the Fourier coefficients of $S(t)$ in (4.9) (cf. [37], Exam. 5.6.1). Take $\mu \in D_{\theta}(\Gamma)$, we have that

$$
\left(\mathrm{V}_{\theta}^{k, \widetilde{k}}(\mu) \circ \boldsymbol{z}\right)(s)=e^{\imath \theta s} \int_{0}^{2 \pi}\left(\widehat{G}_{\theta}^{k}(s, t)-\widehat{G}_{\theta}^{\widetilde{k}}(s, t)\right) e^{-\imath \theta t}(\mu \circ \boldsymbol{z})(t)\left\|\boldsymbol{z}^{\prime}(t)\right\| d t
$$

as a Lebesgue integral. Furthermore, one has

$$
\widehat{G}_{\theta}^{k}(s, t)-\widehat{G}_{\theta}^{\widetilde{k}}(s, t)=S(t-s)\left(J_{\theta}^{k}(s, t)-J_{\theta}^{\widetilde{k}}(s, t)\right)+\left(R_{\theta}^{k}(s, t)-R_{\theta}^{\widetilde{k}}(s, t)\right) .
$$

Employing Lemma 4.11, Theorem 4.10 and ([1], Eq. 9.1.13) we see that the second term of the right-hand side of (4.11) gives rise to a bounded operator from $H^{s}[0,2 \pi]$ to $H^{s+p}[0,2 \pi]$ for any $p>0$. On the other hand, the first term in the right-hand side of (4.11) may be decomposed as

$$
S(t-s)\left(J_{\theta}^{k}(s, t)-J_{\theta}^{\widetilde{k}}(s, t)\right)=\left(|\sin (t-s)|^{2} S(t-s)\right)\left(\frac{J_{\theta}^{k}(s, t)-J_{\theta}^{\widetilde{k}}(s, t)}{|\sin (t-s)|^{2}}\right) .
$$

One can see (cf. [1], Eq. 9.1.12) that the term $\left(J_{\theta}^{k}(s, t)-J_{\theta}^{\widetilde{k}}(s, t)\right)|\sin (t-s)|^{-2}$ belongs to $\mathcal{C}^{\infty}(\mathbb{R} \times \mathbb{R})$, whereas the term $|\sin (t-s)|^{2} S(t-s)$ give rise to an operator of order -3 . In fact, its Fourier transform is

$$
-\frac{1}{2 \pi} \int_{0}^{2 \pi} \sin (t)^{2} \log \left|2 \sin \frac{t}{2}\right| e^{\imath n t} d t=-\frac{1}{2 \pi} \int_{0}^{2 \pi} \log \left|2 \sin \frac{t}{2}\right|\left(e^{\imath(n+2) t}+e^{\imath(n-2) t}-2 e^{\imath(n) t}\right) d t \lesssim n^{-3},
$$

where the last equality follows from ([37], Exam. 5.6.1). Finally, define

$$
\widehat{\mathrm{V}}_{\theta}^{k, \widetilde{k}}(\mu)(s):=e^{-\imath \theta s} \mathrm{~V}_{\theta}^{k, \widetilde{k}}(\mu) \circ \boldsymbol{z}(s) .
$$

Then,

$$
\left\|\mathrm{V}_{\theta}^{k, \widetilde{k}}(\mu)\right\|_{H_{\theta}^{s}(\Gamma)} \cong\left\|\mathrm{V}_{\theta}^{k, \widetilde{k}}(\mu) \circ \boldsymbol{z}\right\|_{H_{\theta}^{s}[0,2 \pi]}=\left\|\widehat{\mathrm{V}}_{\theta}^{k, \widetilde{k}}(\mu)(s)\right\|_{H^{s}[0,2 \pi]} .
$$

We may now bound the last term in (4.12) by Theorem 4.10:

$$
\left\|\widehat{\mathrm{V}}_{\theta}^{k, \widetilde{k}}(\mu)\right\|_{H^{s+3}[0,2 \pi]} \lesssim\|\mu\|_{H_{\theta}^{s}(\Gamma)} .
$$

The proof is completed by the density of $\mathcal{D}_{\theta}(\Gamma)$ in the corresponding Sobolev space.

For the hyper-singular BIO, a similar result requires a technical lemma. To this end, let us define the tangential curl operator:

$$
\operatorname{curl}_{\Gamma} \varphi:=\frac{1}{\|\dot{\boldsymbol{z}}(t)\|_{\mathbb{R}^{2}}} \frac{\mathrm{d}}{\mathrm{d} t}(\varphi \circ \boldsymbol{z})(t)
$$

for any $\varphi \in \mathcal{D}_{\theta}(\Gamma)$ and where $\boldsymbol{z}$ is a suitable (arbitrary) parametrization of $\Gamma$.

Lemma 4.13. Let $k$ and $\Gamma$ satisfy Assumptions 4.2 and 4.4 for $r=0$, respectively, and let $\lambda$ and $\varphi$ belong to $\mathcal{D}_{\theta}(\Gamma)$. Then,

$$
\left\langle\mathrm{W}_{\theta}^{k}(\lambda), \varphi\right\rangle_{\Gamma}=\left\langle\mathrm{V}_{\theta}^{k}\left(\operatorname{curl}_{\Gamma} \lambda\right), \operatorname{curl}_{\Gamma} \varphi\right\rangle_{\Gamma}+\left\langle\widehat{\mathrm{V}}_{\theta}^{k}(\lambda), \varphi\right\rangle_{\Gamma}
$$

where $\langle\cdot, \cdot\rangle_{\Gamma}$ represents the duality product between $H_{\theta}^{s}(\Gamma)$ and $H_{\theta}^{-s}(\Gamma)$ for any $s>0$ and $\bigvee_{\theta}^{k}$ is the extension by density of the operator given by

$$
\left\langle\widehat{V}_{\theta}^{k}(\lambda), \varphi\right\rangle_{\Gamma}:=-k^{2} \int_{\Gamma} \int_{\Gamma} \boldsymbol{n}(\boldsymbol{x}) \cdot \boldsymbol{n}(\boldsymbol{y}) G_{\theta}^{k}(\boldsymbol{x}, \boldsymbol{y}) \lambda(\boldsymbol{y}) \bar{\varphi}(\boldsymbol{x}) \mathrm{d} \boldsymbol{y} \mathrm{d} \boldsymbol{x} .
$$


Proof. Notice that for $\lambda, \varphi$ in $\mathcal{D}_{\theta}(\Gamma)$, it holds that

$$
\left\langle\operatorname{curl}_{\Gamma} \lambda, \varphi\right\rangle_{\Gamma}=\int_{0}^{2 \pi} \frac{\mathrm{d}(\lambda \circ \boldsymbol{z})(t)}{\mathrm{d} t} \overline{(\varphi \circ \boldsymbol{z})}(t) \mathrm{d} t=-\int_{0}^{2 \pi} \frac{\mathrm{d} \overline{(\varphi \circ \boldsymbol{z})}(t)}{\mathrm{d} t}(\lambda \circ \boldsymbol{z})(t) \mathrm{d} t,
$$

where the border terms cancel each other out due to the quasi-periodicity of $\lambda$ and $\varphi$. Hence, the result for quasi-periodic functions follows verbatim from the standard case ( $c f$. [42], Thm. 6.15).

Corollary 4.14. Under the assumptions of Proposition 4.12, consider $\mathrm{W}_{\theta}^{k}$, and $\mathrm{W}_{\theta}^{\widetilde{k}}$, the hyper-singular operators defined as in (4.4) and where again we drop the $\Gamma$ subscript. The operator $\mathrm{W}_{\theta}^{k, \widetilde{k}}:=\mathrm{W}_{\theta}^{k}-\mathrm{W}_{\theta}^{\widetilde{k}}$ can be extended to

$$
\mathrm{W}_{\theta}^{k, \widetilde{k}}: H_{\theta}^{s}(\Gamma) \rightarrow H_{\theta}^{s+1}(\Gamma),
$$

as a bounded linear operator for every $s \in \mathbb{R}$.

Proof. Let $\lambda, \varphi$ in $\mathcal{D}_{\theta}(\Gamma)$. By Lemma 4.13, we have that

$$
\left\langle\mathrm{W}_{\theta}^{k, \widetilde{k}}(\lambda), \varphi\right\rangle_{\Gamma}=\left\langle\mathrm{V}_{\theta}^{k, \widetilde{k}}\left(\operatorname{curl}_{\Gamma} \lambda\right), \operatorname{curl}_{\Gamma} \varphi\right\rangle_{\Gamma}+\left\langle\left(\breve{\mathrm{V}}_{\theta}^{k}-\breve{\mathrm{V}}_{\theta}^{\widetilde{k}}\right)(\lambda), \varphi\right\rangle_{\Gamma}
$$

Using Proposition 4.12, one obtains

$$
\left|\left\langle\mathrm{W}_{\theta}^{k, \widetilde{k}}(\lambda), \varphi\right\rangle_{\Gamma}\right| \lesssim\left\|\operatorname{curl}_{\Gamma} \lambda\right\|_{H_{\theta}^{s-1}(\Gamma)}\left\|\operatorname{curl}_{\Gamma} \varphi\right\|_{H_{\theta}^{-s-2}(\Gamma)}+\|\lambda\|_{H_{\theta}^{s}(\Gamma)}\|\varphi\|_{H_{\theta}^{-s-1}(\Gamma)} .
$$

Where the inequality for the second term of the right-hand side is obtained using that both $\left(\mathrm{V}_{\theta}^{k}, \mathrm{~V}_{\theta}^{\widetilde{k}}\right)$ are operators of order -1 by Theorem 4.10 and ([37], Exam. 5.6.1). Then, $\operatorname{since} \operatorname{curl}_{\Gamma}$ is a first-order differential operator, it holds that

$$
\left|\left\langle\mathrm{W}_{\theta}^{k, \widetilde{k}}(\lambda), \varphi\right\rangle_{\Gamma}\right| \lesssim\|\lambda\|_{H_{\theta}^{s}(\Gamma)}\|\varphi\|_{H_{\theta}^{-s-1}(\Gamma)} .
$$

The result then follows by a duality argument and recalling the density of $\mathcal{D}_{\theta}(\Gamma)$ in our quasi-periodic Sobolev spaces.

We now consider Dirichlet traces of the double layer potential and its adjoint, defined in Section 4.2 as the principal value integrals

$$
\begin{gathered}
\left(\mathrm{K}_{\theta}^{\prime k}(\mu) \circ \boldsymbol{r}\right)(s)=\int_{0}^{2 \pi} \mathcal{K}_{\theta}^{\prime k}(s, t)(\mu \circ \boldsymbol{z})(t)\|\dot{\boldsymbol{z}}(t)\| \mathrm{d} t, \\
\left(\mathrm{~K}_{\theta}^{k}(\lambda) \circ \boldsymbol{r}\right)(s)=\int_{0}^{2 \pi} \mathcal{K}_{\theta}^{k}(s, t)(\lambda \circ \boldsymbol{z})(t)\|\dot{\boldsymbol{z}}(t)\| \mathrm{d} t,
\end{gathered}
$$

for which we have dropped the $\Gamma$ index momentarily, and where the kernels are given by ([14], Sect. 3):

$$
\begin{aligned}
\mathcal{K}_{\theta}^{\prime k}(s, t) & :=-\frac{\imath k}{4} \sum_{j=-\infty}^{\infty}\left(\frac{H_{1}^{(1)}\left(k\left\|\boldsymbol{z}(s)+2 \pi j \boldsymbol{e}_{\mathbf{1}}-\boldsymbol{z}(t)\right\|\right)}{\left\|\boldsymbol{z}(s)+2 \pi j \boldsymbol{e}_{\mathbf{1}}-\boldsymbol{z}(t)\right\|} e^{-\imath 2 \pi j \theta}\right. \\
& \left.\times\left(\boldsymbol{z}(s)+2 \pi j \boldsymbol{e}_{\mathbf{1}}-\boldsymbol{z}(t)\right) \cdot \boldsymbol{n}(\boldsymbol{z}(s))\right) \\
\mathcal{K}_{\theta}^{k}(s, t) & :=\frac{\imath k}{4} \sum_{j=-\infty}^{\infty}\left(\frac{H_{1}^{(1)}\left(k\left\|\boldsymbol{z}(s)+2 \pi j \boldsymbol{e}_{\mathbf{1}}-\boldsymbol{z}(t)\right\|\right)}{\left\|\boldsymbol{z}(s)+2 \pi j \boldsymbol{e}_{\mathbf{1}}-\boldsymbol{z}(t)\right\|} e^{-\imath 2 \pi j \theta}\right. \\
& \left.\times\left(\boldsymbol{z}(s)+2 \pi j \boldsymbol{e}_{\mathbf{1}}-\boldsymbol{z}(t)\right) \cdot \boldsymbol{n}(\boldsymbol{z}(t))\right)
\end{aligned}
$$


where $\boldsymbol{n}$ denotes the unitary normal vector exterior to $\Omega$ - recall that $\Gamma:=\partial^{\mathcal{G}} \Omega$. These can be written as ([1], Eq. 9.1.11)

$$
\begin{aligned}
& \mathcal{K}_{\theta}^{\prime k}(s, t)=S_{1}(t-s) J_{1, \theta}^{k}(s, t)+R_{1, \theta}^{k}(s, t) \\
& \mathcal{K}_{\theta}^{k}(s, t)=S_{1}(t-s) J_{2, \theta}^{k}(s, t)+R_{2, \theta}^{k}(s, t),
\end{aligned}
$$

wherein

$$
\begin{aligned}
& S_{1}(t-s):=-\frac{1}{2 \pi} \log \left(2 \sin \left(\frac{1}{2}|t-s|\right)\right)|\sin (t-s)|^{2}, \\
& J_{1, \theta}^{k}(s, t):=-k \sum_{j=-\infty}^{\infty}\left(\frac{J_{1}\left(k\left\|\boldsymbol{z}(s)+2 \pi j \boldsymbol{e}_{\mathbf{1}}-\boldsymbol{z}(t)\right\|\right)}{\left\|\boldsymbol{z}(s)+2 \pi j \boldsymbol{e}_{\mathbf{1}}-\boldsymbol{z}(t)\right\|} e^{-\imath 2 \pi j \theta}\right. \\
&\left.\quad \times \frac{\left(\boldsymbol{z}(s)+2 \pi j \boldsymbol{e}_{\mathbf{1}}-\boldsymbol{z}(t)\right) \cdot \boldsymbol{n}(\boldsymbol{z}(s))}{|\sin (t-s)|^{2}} \chi_{\epsilon}(t-2 \pi j-s)\right), \\
& J_{2, \theta}^{k}(s, t):=k \sum_{j=-\infty}^{\infty}\left(\frac{J_{1}\left(k\left\|\boldsymbol{z}(s)+2 \pi j \boldsymbol{e}_{\mathbf{1}}-\boldsymbol{z}(t)\right\|\right)}{\left\|\boldsymbol{z}(s)+2 \pi j \boldsymbol{e}_{\mathbf{1}}-\boldsymbol{z}(t)\right\|} e^{-\imath 2 \pi j \theta}\right. \\
& \times \\
&\left.\times \frac{\left(\boldsymbol{z}(s)+2 \pi j \boldsymbol{e}_{\mathbf{1}}-\boldsymbol{z}(t)\right) \cdot \boldsymbol{n}(\boldsymbol{z}(t))}{|\sin (t-s)|^{2}} \chi_{\epsilon}(t-2 \pi j-s)\right),
\end{aligned}
$$

and

$$
\begin{aligned}
& R_{1, \theta}^{k}(s, t):=\mathcal{K}_{\theta}^{\prime k}(s, t)-S_{1}(t-s) J_{1, \theta}^{k}(s, t), \\
& R_{2, \theta}^{k}(s, t):=\mathcal{K}_{\theta}^{k}(s, t)-S_{1}(t-s) J_{2, \theta}^{k}(s, t) .
\end{aligned}
$$

As in the proof of Proposition 4.12, we have that $\left|S_{1, n}\right| \lesssim n^{-3}$, whence, arguing as in Proposition 4.12, we have the following result.

Proposition 4.15. For $k$ and $\Gamma$ as in Assumptions 4.2 and 4.4 with $r=\infty$, respectively, and for any $s \in \mathbb{R}$, it holds that

$$
\mathrm{K}_{\theta}^{\prime k}: H_{\theta}^{s}(\Gamma) \rightarrow H_{\theta}^{s+3}(\Gamma), \quad \mathrm{K}_{\theta}^{k}: H_{\theta}^{s}(\Gamma) \rightarrow H_{\theta}^{s+3}(\Gamma),
$$

are bounded and linear operators.

As in the case of the weakly and hyper-singular BIO, we define:

$$
\mathrm{K}_{\theta}^{\prime k, \widetilde{k}}:=\mathrm{K}_{\theta}^{\prime k}-\mathrm{K}_{\theta}^{\prime \widetilde{k}}, \quad \mathrm{~K}_{\theta}^{k, \widetilde{k}}:=\mathrm{K}_{\theta}^{k}-\mathrm{K}_{\theta}^{\widetilde{k}}
$$

Finally, we obtain our compactness result.

Proposition 4.16. Let $k$ and $\widetilde{k}$ satisfy Assumption 4.2, let $\Gamma$ be as in Assumption 4.4 with $r=\infty$. Then, for $s \in \mathbb{R}$, the following operators

$$
\begin{aligned}
& \mathrm{V}_{\theta}^{k, \widetilde{k}}: H_{\theta}^{s}(\Gamma) \rightarrow H_{\theta}^{s+3-\epsilon}(\Gamma), \quad \mathrm{W}_{\theta}^{k, \widetilde{k}}: H_{\theta}^{s}(\Gamma) \rightarrow H_{\theta}^{s+1-\epsilon}(\Gamma), \\
& \mathrm{K}_{\theta}^{k, \widetilde{k}}: H_{\theta}^{s}(\Gamma) \rightarrow H_{\theta}^{s+3-\epsilon}(\Gamma), \quad \mathrm{K}_{\theta}^{\prime k, \widetilde{k}}: H_{\theta}^{s}(\Gamma) \rightarrow H_{\theta}^{s+3-\epsilon}(\Gamma),
\end{aligned}
$$

are compact for every $\epsilon>0$. 
Proof. The result is direct from the mapping properties shown and Theorem 4.8.

Lastly, we require the compactness of the operator resulting from taking traces of the single and double layer operators acting on densities lying on a boundary $\Gamma_{1}$ over another $x_{1}$-periodic curve, say $\Gamma_{2}$, that does not intersect with $\Gamma_{1}$. Let us denote by $\gamma_{d}^{2}, \gamma_{n}^{2}$ Dirichlet and Neumann traces over $\Gamma_{2}$, respectively. Then, by an application of Lemma 4.11, Theorem 4.8 and Theorem 4.10, we obtain the following result.

Proposition 4.17. Let $k$ satisfy Assumption 4.2. If $\Gamma_{1}$ and $\Gamma_{2}$ are periodic boundaries of class $\mathcal{C}^{\infty}$, then the application of the following traces to the layer potentials:

$$
\begin{gathered}
\gamma_{\mathrm{D}}^{2} \mathrm{SL}_{\theta, \Gamma_{1}}^{k}: H_{\theta}^{s_{1}}\left(\Gamma_{1}\right) \rightarrow H_{\theta}^{s_{2}}\left(\Gamma_{2}\right), \quad \gamma_{\mathrm{N}}^{2} \mathrm{SL}_{\theta, \Gamma_{1}}^{k}: H_{\theta}^{s_{1}}\left(\Gamma_{1}\right) \rightarrow H_{\theta}^{s_{2}}\left(\Gamma_{2}\right), \\
\gamma_{\mathrm{D}}^{2} \mathrm{DL}_{\theta, \Gamma_{1}}^{k}: H_{\theta}^{s_{1}}\left(\Gamma_{1}\right) \rightarrow H_{\theta}^{s_{2}}\left(\Gamma_{2}\right), \quad \gamma_{\mathrm{N}}^{2} \mathrm{DL}_{\theta, \Gamma_{1}}^{k}: H_{\theta}^{s_{1}}\left(\Gamma_{1}\right) \rightarrow H_{\theta}^{s_{2}}\left(\Gamma_{2}\right),
\end{gathered}
$$

are compact operators for any choice of $s_{1}, s_{2} \in \mathbb{R}$. The result holds regardless of the direction from which the traces are taken.

Remark 4.18. For the main results in this section, we have assumed the interfaces to be of class $\mathcal{C}^{\infty}$. While this simplifies the analysis, we could obtain similar results with less stringent regularity requirements. Consider $k$ and $\widetilde{k}$ satisfying Assumption 4.2 and $\Gamma$ as in Assumption 4.4 with $r \in[1, \infty)$, and the weakly-singular operator $V_{\theta}^{k}$ (where we have omitted the $\Gamma$ sub-index momentarily). The expression in (4.8) still holds for the kernel of $V_{\theta}^{k}$, but $R_{\theta}^{k}$ and $J_{\theta}^{k}$ would be only of class $\mathcal{C}^{r, 1}$, instead of arbitrarily smooth. Corollary 6.1.1 and Lemma 6.1.3 in [37] imply the same results of Propositions 4.12 and 4.16 for $s$ in a range limited by $r$.

Remark 4.19. As aforementioned, we have limited ourselves to extending the classical mapping results of the BIOs to the context of quasi-periodic spaces. For the classical result see, for example, ([12], Thm. 2.1).

\subsection{Boundary integral formulation}

We recall the notation and geometry configuration introduced in Section 3, that is:

1. $u^{\text {(inc) }}$ denotes a plane incident wave with wavenumber $k_{0}$, which is assumed to be quasi-periodic with shift $\theta \in[0,1)$.

2. $\left\{\Gamma_{i}\right\}_{i=1}^{M}$ denotes a set of $M \in \mathbb{N}$ non-intersecting periodic boundaries of class $\mathcal{C}^{r, 1}$, with $r \in[1, \infty]$, ordered downwards.

3. $\left\{\Omega_{i}\right\}_{i=0}^{M}$ denotes a set of $M+1$ open domains, ordered downwards with boundaries

$$
\partial^{\mathcal{G}} \Omega_{0}=\Gamma_{1}, \quad \partial^{\mathcal{G}} \Omega_{i}=\Gamma_{i} \cup \Gamma_{i+1} \quad \forall i \in\{1, \ldots, M-1\}, \quad \partial^{\mathcal{G}} \Omega_{M}=\Gamma_{M} .
$$

4. $\left\{\eta_{i}\right\}_{i=1}^{M}$ denotes a parameter set such that the wavenumber in $\Omega_{i}$ is given by $k_{i}=\eta_{i} k_{0}$ for $i \in\{1, \ldots, M\}$.

Assumption 4.20. For a given shift, $\theta$, the wavenumber $k_{0}$ and the parameters $\left\{\eta_{i}\right\}_{i=1}^{M}$ are such that neither $k_{0}$ nor the wavenumbers $k_{i}=\eta_{i} k_{0}$ are cut-off frequencies.

Following the notation of Problem 3.1, the scattered field - defined as the total field $u^{\text {(tot) }}$ minus the incident field $u^{(\text {inc })}-$ is written as

$$
u^{(\mathrm{sc})}:=u_{i} \text { in } \Omega_{i}, \text { for } i \in\{0, \ldots, M\} .
$$

Under Assumption 4.20, we make the following representation Ansatz for the scattered field:

$$
u^{(\mathrm{sc})}= \begin{cases}\mathrm{SL}_{\theta, \Gamma_{1}}^{k_{0}}\left(\mu_{1}\right)-\mathrm{DL}_{\theta, \Gamma_{1}}^{k_{0}}\left(\lambda_{1}\right) & \text { in } \Omega_{0}, \\ \operatorname{SL}_{\theta, \Gamma_{i}}^{k_{i}}\left(\mu_{i}\right)-\mathrm{DL}_{\theta, \Gamma_{i}}^{k_{i}}\left(\lambda_{i}\right) & \text { in } \Omega_{i}, \text { for } i \in\{1, \ldots, M-1\} \\ +\mathrm{SL}_{\theta, \Gamma_{i+1}}^{k_{i}}\left(\mu_{i+1}\right)-\mathrm{DL}_{\theta, \Gamma_{i+1}}^{k_{i}}\left(\lambda_{i+1}\right) & \text { in } \Omega_{M}, \\ \mathrm{SL}_{\theta, \Gamma_{M}}^{k_{M}}\left(\mu_{M}\right)-\mathrm{DL}_{\theta, \Gamma_{M}}^{k_{M}}\left(\lambda_{M}\right) & \end{cases}
$$


where, for each $i \in\{1, \ldots, M\}$, the boundary data $\lambda_{i}$ and $\mu_{i}$ are assumed to belong to $H_{\theta}^{s}\left(\Gamma_{i}\right)$ for some possibly different values of $s \in \mathbb{R}$, i.e., $s$ may be different for each boundary datum. $\mathrm{SL}_{\theta, \Gamma_{i}}^{k_{j}}$ and $\mathrm{DL}_{\theta, \Gamma_{i}}^{k_{j}}$ are, respectively, the single and double layer potentials of wavenumber $k_{j}$ on $\Gamma_{i}$.

As shorthand, in what follows, we denote, for each $i \in\{1, \ldots, M\}$,

$$
\Lambda_{i}:=\left(\lambda_{i}, \mu_{i}\right)^{t}, \quad \mathbf{L}_{\theta, \Gamma_{i}}^{k} \Lambda_{i}:=\mathrm{SL}_{\theta, \Gamma_{i}}^{k}\left(\mu_{i}\right)-\mathrm{DL}_{\theta, \Gamma_{i}}^{k}\left(\lambda_{i}\right)
$$

where $\lambda_{i}$ and $\mu_{i}$ are defined over $\Gamma_{i}$. For $s_{1}, s_{2} \in \mathbb{R}$, we define the Cartesian product spaces:

$$
\mathcal{V}_{\theta, \Gamma_{i}}^{s_{1}, s_{2}}:=H_{\theta}^{s_{1}}\left(\Gamma_{i}\right) \times H_{\theta}^{s_{2}}\left(\Gamma_{i}\right) \quad \text { for } i=0, \ldots, M, \quad \text { and } \quad \mathcal{V}_{\theta}^{s_{1}, s_{2}}:=\prod_{i=1}^{M} \mathcal{V}_{\theta, \Gamma_{i}}^{s_{1}, s_{2}}
$$

where all of these spaces are equipped with their natural graph inner products. For each $i \in\{1, \ldots, M\}$, let us define the following operators:

$$
\mathrm{A}_{i} \Lambda_{i}:=\left(\begin{array}{l}
-\mathrm{K}_{\theta, \Gamma_{i}}^{k_{i-1}, k_{i}}\left(\lambda_{i}\right)+\mathrm{V}_{\theta, \Gamma_{i}, k_{i}}^{k_{i-1}, k_{i}}\left(\mu_{i}\right) \\
\mathrm{W}_{\theta, \Gamma_{i}}^{k_{i-1}, k_{i}}\left(\lambda_{i}\right)+\mathrm{K}_{\theta, \Gamma_{i}}^{k_{i-1}, k_{i}}\left(\mu_{i}\right)
\end{array}\right)
$$

corresponding to self-interactions between the potentials defined over each $\Gamma_{i}$ with themselves. Analogously, for $i, j \in\{1, \ldots, M\}$, we define the following operators:

$$
\mathrm{B}_{i, j} \Lambda_{j}:=\left\{\begin{array}{cl}
\left(\begin{array}{cc}
-\gamma_{\mathrm{D}}^{i} \mathrm{DL}_{\theta, \Gamma_{j}}^{k_{\min \{i, j\}}}\left(\lambda_{j}\right)+\gamma_{\mathrm{D}}^{i} \mathrm{SL}_{\theta, \Gamma_{j}\{i, j\}}^{k_{\min }}\left(\mu_{j}\right) \\
-\gamma_{\mathrm{N}}^{i} \mathrm{DL}_{\theta, \Gamma_{j}}^{k_{\min }\{i, j\}}\left(\lambda_{j}\right)+\gamma_{\mathrm{N}}^{i} \mathrm{SL}_{\theta, \Gamma_{j}}^{k_{\min }\{i, j\}}\left(\mu_{j}\right)
\end{array}\right) & \text { if }|i-j|=1 \\
\mathbf{0} & \text { a.o.c. }
\end{array}\right.
$$

corresponding to interactions between potentials defined over $\Gamma_{i}$ with those defined over $\Gamma_{j}$.

Proposition 4.21. Let Assumption 4.20 hold and let interfaces $\left\{\Gamma_{i}\right\}_{i=1}^{M}$ be of class $\mathcal{C}^{\infty}$. Then, the selfinteraction operators defined in (4.15)

$$
\mathrm{A}_{i}: \mathcal{V}_{\theta, \Gamma_{i}}^{s_{1}, s_{2}} \rightarrow \mathcal{V}_{\theta, \Gamma_{i}}^{s_{1}, s_{2}}
$$

are compact operators for any $s_{1}, s_{2} \in \mathbb{R}$ with $s_{2}<s_{1}<s_{2}+2$. Furthermore, the cross-interaction operators (4.16)

$$
\mathrm{B}_{i, j}: \mathcal{V}_{\theta, \Gamma_{j}}^{s_{1}, s_{2}} \rightarrow \mathcal{V}_{\theta, \Gamma_{i}}^{s_{1}, s_{2}}
$$

are compact for any choice of $s_{1}, s_{2} \in \mathbb{R}$.

Proof. The first result is directly found using Proposition 4.16, whereas the second one follows from Proposition 4.17.

With the above definitions and using the jump properties of the BIOs, it holds that

$$
\left[\gamma u^{(\mathrm{sc})}\right]_{\Gamma_{i}}=\mathrm{B}_{i, i-1} \Lambda_{i-1}+\left(\mathrm{A}_{i}-\mathrm{I}_{i}\right) \Lambda_{i}-\mathrm{B}_{i, i+1} \Lambda_{i+1}
$$

for each $i \in\{1, \ldots, M\}$, where $\mathrm{I}_{i}$ corresponds to the identity map over $\mathcal{V}_{\theta, \Gamma_{j}}^{s_{1}, s_{2}}$, with $s_{1}, s_{2} \in \mathbb{R}$. We now introduce the following operator matrix over $\mathcal{V}_{\theta}^{s_{1}, s_{2}}$ :

$$
\mathcal{M}:=\left(\begin{array}{ccccccc}
\mathrm{A}_{1}-\mathrm{I}_{1} & -\mathrm{B}_{1,2} & 0 & 0 & 0 & \ldots & 0 \\
\mathrm{~B}_{2,1} & \mathrm{~A}_{2}-\mathrm{I}_{2} & -\mathrm{B}_{2,3} & 0 & 0 & \ldots & 0 \\
\vdots & \vdots & \vdots & \vdots & \vdots & \vdots & \vdots \\
0 & 0 & \ldots & 0 & \mathrm{~B}_{M-1, M-2} & \mathrm{~A}_{M-1}-\mathrm{I}_{M-1} & \mathrm{~B}_{M-1, M} \\
0 & 0 & \ldots & 0 & 0 & \mathrm{~B}_{M, M-1} & \mathrm{~A}_{M}-\mathrm{I}_{M}
\end{array}\right) .
$$

Imposing the boundary conditions of Problem 3.1 to $u^{(\mathrm{sc})}$ leads to the following system of BIEs. 
Problem 4.22. Let Assumption 4.20 hold and let $s \in \mathbb{R}$. Set $s_{1}=s+\frac{1}{2}$ and $s_{2}=s-\frac{1}{2}$. We seek $\boldsymbol{\Lambda} \in \mathcal{V}_{\theta}^{s_{1}, s_{2}}$ such that

$$
\mathcal{M} \Lambda=\left(\begin{array}{c}
-\gamma^{e, 1} u^{(\mathrm{inc})} \\
0 \\
\vdots \\
0
\end{array}\right)
$$

where $\mathcal{M}$ corresponds to the operator matrix in (4.18) and $\boldsymbol{\gamma}^{e, 1}$ corresponds to the exterior trace vector on $\Gamma_{1}$.

In order to ensure the well-posedness of Problem 4.22, we introduce the following set of auxiliary problems.

Problem 4.23 (Auxiliary problems). We seek $\left\{v_{i}\right\}_{i=1}^{M}$ such that $v_{i} \in H_{\theta, \text { loc }}^{1}\left(\mathcal{G} \backslash \Gamma_{i}\right)$ and

$$
\begin{aligned}
-\left(\Delta+k_{i}^{2}\right) v_{i}(\boldsymbol{x}) & =0 \quad \text { in }\left(\Omega_{i-1} \cup \bigcup_{j=0}^{i-2} \overline{\Omega_{j}} \mathcal{G}\right) \cap\left\{\boldsymbol{x} \in \mathcal{G}:\left|x_{2}\right|<H\right\}, \\
-\left(\Delta+k_{i-1}^{2}\right) v_{i}(\boldsymbol{x}) & =0 \quad \text { in } \Omega_{i} \cup\left(\bigcup_{j=i+1}^{M} \overline{\Omega_{j} \mathcal{G}}\right) \cap\left\{\boldsymbol{x} \in \mathcal{G}:\left|x_{2}\right|<H\right\}, \\
{\left[\gamma v_{i}\right]_{\Gamma_{i}} } & =0 \quad \text { on } \Gamma_{i}, \\
v_{i}(\boldsymbol{x}) & =\sum_{j \in \mathbb{Z}} v_{j}^{(i)} e^{\imath\left(\beta_{j}^{(i)}\left(x_{2}-H\right)+j_{\theta} x_{1}\right)} \quad \text { for all } x_{2} \geq H, \\
v_{i}(\boldsymbol{x}) & =\sum_{j \in \mathbb{Z}} v_{j}^{(i)} e^{\imath\left(\beta_{j}^{(i-1)}\left(x_{2}+H\right)+j_{\theta} x_{1}\right)} \quad \text { for all } x_{2} \leq-H,
\end{aligned}
$$

with

$$
\beta_{j}^{(i)}:=\left\{\begin{array}{ll}
\sqrt{k_{i}^{2}-j_{\theta}^{2}} & \text { if } k_{i}^{2}-j_{\theta}^{2} \geq 0 \\
\imath \sqrt{j_{\theta}^{2}-k_{i}^{2}} & \text { if } k_{i}^{2}-j_{\theta}^{2}<0
\end{array} \quad \forall j \in \mathbb{Z}\right.
$$

for each $i \in\{1, \ldots, M\}$, and where $H>0$ is as in Section 3.1 and $\left\{k_{i}\right\}_{i=0}^{M}$ are the wavenumbers in each $\left\{\Omega_{i}\right\}_{i=0}^{M}$, as introduced in Section 3.

By a similar analysis to the one presented in ([41], Sect. 3.4), each interface $\Gamma_{i}, i \in\{1, \ldots, M\}$, potentially adds a countable set of wavenumbers, $k_{0}$, such that Problem 4.23 is unsolvable. This justifies the following Assumption - recall $k_{i}=\eta_{i} k_{0}$ for all $i \in\{1, \ldots, M\}$.

Assumption 4.24. Given $\left\{\eta_{i}\right\}_{i=1}^{M}$, the wavenumber $k_{0}$ is such that the auxiliary Problem 4.23 has only one solution $\left\{v_{i}\right\}_{i=1}^{M}$ given by $v_{i}:=0$ for all $i \in\{1, \ldots, M\}$.

Assumption 4.24 will force us to discard yet more wavenumbers, but the set of wavenumbers neglected by Assumptions 3.2 and 4.24 is still countable.

Theorem 4.25. Let parameters $k_{0}$ and $\left\{\eta_{i}\right\}_{i=1}^{M}$ satisfy Assumption 4.20 and let interfaces $\left\{\Gamma_{i}\right\}_{i=1}^{M}$ be periodic boundaries of class $\mathcal{C}^{\infty}$. Under Assumptions 3.2 and 4.24, Problem 4.22 is well posed for any $s \in \mathbb{R}$.

Proof. Note that the operator matrix $\mathcal{M}$ may be written as

$$
\mathcal{M}=\left(\begin{array}{ccccccc}
\mathrm{A}_{1} & -\mathrm{B}_{1,2} & 0 & 0 & 0 & \ldots & 0 \\
\mathrm{~B}_{2,1} & \mathrm{~A}_{2} & -\mathrm{B}_{2,3} & 0 & 0 & \ldots & 0 \\
\vdots & \vdots & \vdots & \vdots & \vdots & \vdots & \vdots \\
0 & 0 & \ldots & 0 & \mathrm{~B}_{M-1, M-2} & \mathrm{~A}_{M-1} & \mathrm{~B}_{M-1, M} \\
0 & 0 & \ldots & 0 & 0 & \mathrm{~B}_{M, M-1} & \mathrm{~A}_{M}
\end{array}\right)-\left(\begin{array}{cccccc}
\mathrm{I}_{1} & 0 & 0 & 0 & \ldots & 0 \\
0 & \mathrm{I}_{2} & 0 & 0 & \ldots & 0 \\
\vdots & \vdots & \vdots & \vdots & \vdots & \vdots \\
0 & 0 & \ldots & 0 & \mathrm{I}_{M-1} & 0 \\
0 & 0 & \ldots & 0 & 0 & \mathrm{I}_{M}
\end{array}\right)
$$


By the Fredholm alternative, we need only show uniqueness of Problem 4.22, as the above tridiagonal block is compact by Proposition 4.21. The proof is similar to the one found for the classical problem of scattering by a bounded object in free space (cf. [20], Thm. 3.41).

Let $\boldsymbol{\Lambda} \in \mathcal{V}_{\theta}^{s_{1}, s_{2}}$, with $s_{1}=s+\frac{1}{2}$ and $s_{2}=s-\frac{1}{2}$, be such that $\boldsymbol{M} \boldsymbol{\Lambda}=\mathbf{0}$. We define

$$
\begin{array}{rlrl}
\widetilde{u}_{0}(\boldsymbol{x}) & :=\left(\mathbf{L}_{\theta, \Gamma_{1}}^{k_{0}}\left(\Lambda_{1}\right)\right)(\boldsymbol{x}) & \forall \boldsymbol{x} \in \mathcal{G} \backslash \Gamma_{1}, \\
\widetilde{u}_{i}(\boldsymbol{x}):=\left(\mathbf{L}_{\theta, \Gamma_{i}}^{k_{i}}\left(\Lambda_{i}\right)\right)(\boldsymbol{x})+\left(\mathbf{L}_{\theta, \Gamma_{i+1}}^{k_{i}}\left(\Lambda_{i+1}\right)\right)(\boldsymbol{x}) & \forall \boldsymbol{x} \in \mathcal{G} \backslash\left(\Gamma_{i} \cup \Gamma_{i+1}\right), \forall i \in\{1, \ldots, M-1\}, \\
\widetilde{u}_{m}(\boldsymbol{x}):=\left(\mathbf{L}_{\theta, \Gamma_{m}}^{k_{M}}\left(\Lambda_{m}\right)\right)(\boldsymbol{x}) & \forall \boldsymbol{x} \in \mathcal{G} \backslash \Gamma_{m},
\end{array}
$$

where $\mathbf{L}$ is defined as in (4.14). We further define

$$
\widetilde{u}(\boldsymbol{x}):=\widetilde{u}_{i}(\boldsymbol{x}) \quad \forall \boldsymbol{x} \in \Omega_{i}, \quad \forall i \in\{0, \ldots, M\},
$$

which is well defined in each $\Omega_{i}$. Moreover, $\boldsymbol{M} \boldsymbol{\Lambda}=0$ implies $[\boldsymbol{\gamma} \widetilde{u}]_{\Gamma_{i}}=0$ on each $\Gamma_{i}$, and thus, $\widetilde{u}$ solves the Helmholtz equation with wavenumber $k_{i}$ in each $\Omega_{i}$ and satisfies appropriate radiation conditions at infinity ([5], Sect. 4). Hence, $\widetilde{u}$ solves Problem 3.1, and Assumption 3.2 implies $\widetilde{u} \equiv 0$.

Let us now define the following auxiliary functions:

$$
\widetilde{v}_{i}(\boldsymbol{x}):=\left\{\begin{array}{ll}
\widetilde{u}_{i}(\boldsymbol{x}) & \forall \boldsymbol{x} \in \Omega_{i-1} \cup\left(\bigcup_{j=0}^{i-2} \overline{\Omega_{j} \mathcal{G}}\right) \\
-\widetilde{u}_{i-1}(\boldsymbol{x}) & \forall \boldsymbol{x} \in \Omega_{i} \cup\left(\bigcup_{j=i+1}^{M} \overline{\Omega_{j}} \mathcal{G}\right)
\end{array}, \quad \forall i \in\{1, \ldots, M\} .\right.
$$

It is clear from this definition that

$$
\begin{gathered}
\left(-\Delta-k_{i}^{2}\right) \widetilde{v}_{i}(\boldsymbol{x})=0, \quad \text { in } \Omega_{i-1} \cup\left(\bigcup_{j=0}^{i-2} \overline{\Omega_{j}} \mathcal{G}\right), \\
\left(-\Delta-k_{i-1}^{2}\right) \widetilde{v}_{i}(\boldsymbol{x})=0, \quad \text { in } \Omega_{i} \cup\left(\bigcup_{j=i+1}^{M} \overline{\Omega_{j}} \mathcal{G}\right) .
\end{gathered}
$$

Furthermore, each $\widetilde{v}_{i}$ satisfies the appropriate radiation conditions at infinity. Using the jump relationships of BIOs ([5], Lem. 4.11), we have that

$$
\gamma^{i, e} \widetilde{v}_{i}-\gamma^{i} \widetilde{u}=\Lambda_{i}, \quad \gamma^{i, e} \widetilde{u}+\gamma^{i} \widetilde{v}_{i}=\Lambda_{i}
$$

Since $\widetilde{u} \equiv 0$, we have that

$$
\left[\gamma \widetilde{v}_{i}\right]_{\Gamma_{i}}=\gamma^{i, e} \widetilde{v}_{i}-\gamma^{i} \widetilde{v}_{i}=\gamma^{i, e} \widetilde{v}_{i}-\gamma^{i} \widetilde{u}-\left(\gamma^{i, e} \widetilde{u}+\gamma^{i} \widetilde{v}_{i}\right)=0,
$$

from where it follows that $\left\{\widetilde{v}_{i}\right\}_{i=1}^{M}$ solves Problem 4.23. Assumption 4.24 implies that $\widetilde{v}_{i} \equiv 0$, for all $i$ in $\{1, \ldots, M\}$. Finally, (4.20) implies $\boldsymbol{\Lambda} \equiv 0$ as stated.

Remark 4.26. Theorem 4.25 states that if all interfaces are of arbitrary smoothness, the solution $\boldsymbol{\Lambda}$ is also arbitrarily smooth. This result can be generalized to geometries of limited regularity by following the ideas presented in Remark 4.18, obtaining a solution of limited regularity. 


\section{Spectral Galerkin method}

We now provide a numerical method to approximate solutions of Problem 4.22 along with its corresponding error estimates. We restrict ourselves to cases where the interfaces $\left\{\Gamma_{i}\right\}_{i=1}^{M}$ are periodic boundaries of class $\mathcal{C}^{\infty}$. By Theorem 4.25, the solution is of arbitrary smoothness and a spectral method should converge at a super-algebraic rate (cf. [37], Chap. 9 and [25,26]).

\subsection{Discrete spaces}

Let us define a suitable family of finite dimensional subspaces of $\mathcal{V}_{\theta}^{s_{1}, s_{2}}$. From the definition of quasi-periodic Sobolev spaces, it is natural to consider the following finite dimensional functional spaces over $(0,2 \pi)$ :

$$
\widehat{\mathcal{E}}_{\theta}^{N}:=\operatorname{span}\left\{\widehat{e}_{\theta}^{n}(t):=e^{\imath(n+\theta) t}: n \in\{-N, \ldots, N\}\right\} .
$$

It is clear that $\widehat{\mathcal{E}}_{\theta}^{N} \subset \widehat{\mathcal{E}}_{\theta}^{N+1}$ for all $N \in \mathbb{N}$ and that $\bigcup_{N \in \mathbb{N}} \widehat{\mathcal{E}}_{\theta}^{N}$ is dense in $H_{\theta}^{s}[0,2 \pi]$ for any $s \in \mathbb{R}$. Denoting $\boldsymbol{z}_{i}:(0,2 \pi) \rightarrow \Gamma_{i}$ a parametrization of $\Gamma_{i}$, we define

$$
\begin{gathered}
\widetilde{\mathcal{E}}_{\theta, \Gamma_{i}}^{N}:=\operatorname{span}\left\{\widetilde{e}_{\theta, i}^{n}:=\widehat{e}_{\theta}^{n} \circ \boldsymbol{z}_{i}^{-1},: n \in\{-N, \ldots, N\}\right\}, \\
\mathcal{E}_{\theta, \Gamma_{i}}^{N}:=\operatorname{span}\left\{e_{\theta, i}^{n}:=\left\|\dot{\boldsymbol{z}}_{i} \circ \boldsymbol{z}_{i}^{-1}\right\|_{\mathbb{R}^{2}}^{-1} \widetilde{e}_{\theta, i}^{n}: n \in\{-N, \ldots, N\}\right\} .
\end{gathered}
$$

We can see that $\widetilde{\mathcal{E}}_{\theta, \Gamma_{i}}^{N}$ is the space spanned by finite Fourier basis parametrized on $\Gamma_{i}$ and that $\mathcal{E}_{\theta, \Gamma_{i}}^{N}$ is constructed from the previous space by dividing the basis by the norm of the tangential vector of the corresponding interface. The inclusion of the tangential vector norm in the definition of $\mathcal{E}_{\theta, \Gamma_{i}}^{N}$ allows for a simplified implementation of the discrete problem by factoring out the Jacobian of the parametrization given by $\boldsymbol{z}_{i}$. As before, it is clear that both $\bigcup_{N \in \mathbb{N}} \mathcal{E}_{\theta, \Gamma_{i}}^{N}$ and $\bigcup_{N \in \mathbb{N}} \widetilde{\mathcal{E}}_{\theta, \Gamma_{i}}^{N}$ are dense subspaces of $H_{\theta}^{s}\left(\Gamma_{i}\right)$ for $s \in \mathbb{R}$. Finally, we define the Cartesian product of discrete spaces

$$
\mathcal{E}_{\theta, \Gamma_{i}}^{N}:=\widetilde{\mathcal{E}}_{\theta, \Gamma_{i}}^{N} \times \mathcal{E}_{\theta, \Gamma_{i}}^{N},
$$

whose infinite union on $N$ forms a dense subspace of $\mathcal{V}_{\theta, \Gamma_{i}}^{s_{1}, s_{2}}$ for any pair $s_{1}, s_{2} \in \mathbb{R}$.

\subsection{Discrete problem}

We now consider the Galerkin discretization of Problem 4.22 on the finite dimensional product space

$$
\mathbb{E}_{\theta}^{N}:=\prod_{i=1}^{M} \mathcal{E}_{\theta, \Gamma_{i}}^{N_{i}} \subset \mathcal{V}_{\theta}^{s_{1}, s_{2}} \quad \text { for } \boldsymbol{N}=\left\{N_{i}\right\}_{i=1}^{M} \subset \mathbb{N}, \quad s_{1}, s_{2} \in \mathbb{R} .
$$

Problem 5.1 (Discrete BIEs). Let parameters $k_{0}$ and $\left\{\eta_{i}\right\}_{i=1}^{M}$ satisfy Assumption 4.20 along with interfaces $\left\{\Gamma_{i}\right\}_{i=1}^{M}$ of class $\mathcal{C}^{\infty}$. For some $\boldsymbol{N}=\left\{N_{i}\right\}_{i=1}^{M} \subset \mathbb{N}$, we seek $\boldsymbol{\Lambda}^{\boldsymbol{N}} \in \mathbb{E}_{\theta}^{\boldsymbol{N}}$ such that

$$
\left\langle\mathcal{M} \Lambda^{N}, \Xi^{N}\right\rangle_{\Gamma}=\left\langle\varrho, \Xi^{N}\right\rangle_{\Gamma}, \quad \forall \Xi^{N} \in \mathbb{E}_{\theta}^{N}
$$

where the duality product

$$
\langle\boldsymbol{\Psi}, \boldsymbol{\Xi}\rangle_{\boldsymbol{\Gamma}}:=\sum_{i=1}^{M}\left\langle\Psi_{i}, \Xi_{i}\right\rangle_{\Gamma_{i}} \quad \forall \boldsymbol{\Psi}, \boldsymbol{\Xi} \in \mathcal{V}_{\theta}^{s_{1}, s_{2}}
$$

denotes the sum of two standard duality pairings in $H_{\theta}^{\frac{1}{2}}\left(\Gamma_{i}\right)$ and $H_{\theta}^{-\frac{1}{2}}\left(\Gamma_{i}\right)$, and $\varrho$ accounts for the right-hand side of Problem 4.22. 
Since this is a second-kind BIE, we can deduce a quasi-optimality approximation result for the Galerkin discretization ( $c f$. [38], Thm. 4.2.9), so that in order to establish error convergence rates for the discrete solution, we need to bound those of the best approximation. From the definition of our discrete and continuous spaces, the problem of bounding the best approximation on $\mathcal{V}_{\theta}^{s_{1}, s_{2}}$ is equivalent to that of establishing bounds for the best approximation of an element of $H^{s}[0,2 \pi]$ when approximated by elements of $\widehat{\mathcal{E}}_{\widetilde{\theta}}^{N}$ with $\widetilde{\theta}=0$. This issue was already addressed, for example, in ([37], Thm. 8.2.1). Specifically, for any pair $r_{1}, r_{2} \in \mathbb{R}$ with $r_{2}>r_{1}$ and $f \in H^{r_{2}}[0,2 \pi]$, there holds

$$
\inf _{q \in \widehat{\mathcal{E}}_{\tilde{\theta}}^{N}}\|f-q\|_{H^{r_{1}[0,2 \pi]}} \lesssim N^{r_{1}-r_{2}}\|f\|_{H^{r_{2}[0,2 \pi]}}
$$

Theorem 5.2. Let parameters $k_{0}$ and $\left\{\eta_{i}\right\}_{i=1}^{M}$ satisfy Assumption 4.20 along with interfaces $\left\{\Gamma_{i}\right\}_{i=1}^{M}$ of class $\mathcal{C}^{\infty}$. For $s \geq 0$, let $s_{1}=s+\frac{1}{2}$ and $s_{2}=s-\frac{1}{2}$. Then, under Assumptions 3.2 and 4.24, there exists $\boldsymbol{N}^{\star}=\left\{N_{i}^{\star}\right\}_{i=1}^{M} \subset \mathbb{N}$ such that for any $\boldsymbol{N}=\left\{N_{i}\right\}_{i=1}^{M} \subset \mathbb{N}$ with $N_{i}>N_{i}^{\star}$ for all $i \in\{1, \ldots, M\}$, Problem 5.1 is well posed and it holds that

$$
\left\|\boldsymbol{\Lambda}-\boldsymbol{\Lambda}^{N}\right\|_{\mathcal{V}_{\theta}^{\frac{1}{2},-\frac{1}{2}}} \lesssim\left(\max _{i \in\{1, \ldots, M\}} N_{i}^{-s}\right)\|\boldsymbol{\varrho}\|_{\mathcal{V}_{\theta}^{s_{1}, s_{2}}}
$$

where $\boldsymbol{\Lambda}$ and $\boldsymbol{\Lambda}^{\mathbf{N}}$ are the solutions to Problems 4.22 and 5.1, respectively.

Proof. By ([38], Thm. 4.2.9), together with Proposition 4.21 and the density of $\mathbb{E}_{\theta}^{N}$ in $\mathcal{V}_{\theta}^{\frac{1}{2},-\frac{1}{2}}$, one can ensure the existence of some $\boldsymbol{N}^{\star}=\left\{N_{i}^{\star}\right\}_{i=1}^{M} \subset \mathbb{N}$ such that, for any $\boldsymbol{N}=\left\{N_{i}\right\}_{i=1}^{M} \subset \mathbb{N}$ with $N_{i}>N_{i}^{\star}, i \in\{1, \ldots, M\}$, Problem 5.1 is well posed and it holds that

$$
\left\|\Lambda-\Lambda^{N}\right\|_{\mathcal{V}_{\theta}^{s_{1}, s_{2}}} \lesssim \inf _{\boldsymbol{\Xi}^{N} \in \mathbb{E}_{\theta}^{N}}\left\|\boldsymbol{\Lambda}-\boldsymbol{\Xi}^{N}\right\|_{\mathcal{V}_{\theta}^{s_{1}, s_{2}}},
$$

where $\boldsymbol{\Lambda}$ and $\boldsymbol{\Lambda}^{\boldsymbol{N}}$ are the solutions to Problems 4.22 and 5.1, respectively.

We continue by bounding (5.5). For any $\boldsymbol{\Xi}^{N} \in \mathbb{E}_{\theta}^{N}$, write $\Xi_{i}^{N_{i}}=\left(\xi_{i}^{N_{i}}, \zeta_{i}^{N_{i}}\right)^{t}$ for all $i \in\{1, \ldots, M\}$, so that

$$
\left\|\boldsymbol{\Lambda}-\boldsymbol{\Xi}^{\boldsymbol{N}}\right\|_{\mathcal{V}_{\theta}^{\frac{1}{2},-\frac{1}{2}}}^{2}=\sum_{i=1}^{M}\left\|\lambda_{i}-\xi_{i}^{N_{i}}\right\|_{H_{\theta}^{\frac{1}{2}\left(\Gamma_{i}\right)}}^{2}+\left\|\mu_{i}-\zeta_{i}^{N_{i}}\right\|_{H_{\theta}^{-\frac{1}{2}}\left(\Gamma_{i}\right)}^{2} .
$$

By definition of our continuous and discrete spaces together with (5.4), we see that for all $i \in\{1, \ldots, M\}$, one deduces

$$
\left\|\lambda_{i}-\xi_{i}^{N_{i}}\right\|_{H_{\theta}^{\frac{1}{2}}\left(\Gamma_{i}\right)}^{2} \lesssim N_{i}^{-2 s}\left\|\lambda_{i}\right\|_{H_{\theta}^{s+\frac{1}{2}}\left(\Gamma_{i}\right)}^{2}, \quad\left\|\mu_{i}-\zeta_{i}^{N_{i}}\right\|_{H_{\theta}^{-\frac{1}{2}}\left(\Gamma_{i}\right)}^{2} \lesssim N_{i}^{-2 s}\left\|\mu_{i}\right\|_{H_{\theta}^{s-\frac{1}{2}}\left(\Gamma_{i}\right)}^{2},
$$

where the unspecified constant depends only on $\Gamma_{i}$. Hence, it holds that

$$
\left\|\boldsymbol{\Lambda}-\boldsymbol{\Xi}^{\boldsymbol{N}}\right\|_{\mathcal{V}_{\theta}^{\frac{1}{2},-\frac{1}{2}}}^{2} \lesssim\left(\max _{i \in\{1, \ldots, M\}} N_{i}^{-2 s}\right)\|\boldsymbol{\Lambda}\|_{\mathcal{V}_{\theta}^{s_{1}, s_{2}}}^{2} .
$$

Since the problem is well posed, we obtain

$$
\left\|\boldsymbol{\Lambda}-\boldsymbol{\Xi}^{\boldsymbol{N}}\right\|_{\mathcal{V}_{\theta}^{\frac{1}{2},-\frac{1}{2}}}^{2} \lesssim\left(\max _{i \in\{1, \ldots, M\}} N_{i}^{-2 s}\right)\|\boldsymbol{\varrho}\|_{\mathcal{V}_{\theta}^{s_{1}, s_{2}}}^{2}
$$

where the unspecified constant now also depends on the wavenumbers $\left\{k_{i}\right\}_{i=0}^{M}$. 
Remark 5.3. Theorem 5.2 states that the proposed spectral Galerkin method has a similar performance to the Nyström one. Indeed, if interfaces belong to $\mathcal{C}^{\infty}$, one obtains super-algebraic convergence for both methods (see [46] for Nyström). The super-algebraic convergence rate of the Nyström method for the transmission problem on a bounded object in two dimensions was rigorously proved in [12]. Similar convergence results for quasi-periodic problems using the Nyström scheme are, to the best of our knowledge, not available.

Remark 5.4. It follows from Remark 4.26 that, if the interfaces are of class $\mathcal{C}^{r, 1}$ with $r \in[1, \infty)$, one attains limited convergence orders.

\subsection{Implementation}

We continue with an overview of the procedure employed to compute the approximation $\boldsymbol{\Lambda}^{\boldsymbol{N}}$. For a given $N \in \mathbb{N}$ and $l, m \in \mathbb{Z}$ such that $-N \leq l, m \leq N$, the integrals

$$
I_{l}^{1}:=\int_{0}^{2 \pi} f(t) e^{-\imath l t} \mathrm{~d} t \quad \text { and } \quad I_{l, m}^{2}:=\int_{0}^{2 \pi} \int_{0}^{2 \pi} F(s, t) e^{-\imath l s} e^{\imath m t} \mathrm{~d} t \mathrm{~d} s,
$$

where $f$ and $F$ are smooth periodic and bi-periodic functions, respectively, are computed using the FFT to construct trigonometric interpolations of the corresponding functions (cf. [37], Thm. 8.4.1). In particular, the computation of block matrices $\mathrm{B}_{i, j}$ in (4.18) is performed in this way. Moreover, we have the following result regarding the accuracy of the integral procedure.

Lemma 5.5. For $N^{\prime} \in \mathbb{N}$, let $I_{l}^{1, N^{\prime}}$ denote the approximation of $I_{l}^{1}$ computed through an interpolation of $f$ computed via FFT using $2 N^{\prime}+1$ points. Then, it holds that

$$
\left|I_{l}^{1, N^{\prime}}-I_{l}^{1}\right| \lesssim\left(N^{\prime}\right)^{-p}
$$

for all $l \in \mathbb{Z}$ and $p \in \mathbb{N}$ satisfying $p<N^{\prime}$ and $|l| \leq N^{\prime}$, wherein the unspecified constant depends on $f$ but not on $l$ or $N^{\prime}$. Similarly, let $I_{l, m}^{2}$ denote the analogous approximation of $I_{l, m}^{2, N^{\prime}}$. Then, it holds that

$$
\left|I_{l, m}^{2, N^{\prime}}-I_{l, m}^{2}\right| \lesssim\left(N^{\prime}\right)^{-p}
$$

for all $l, m \in \mathbb{Z}$ and $p \in \mathbb{N}$ satisfying $p<N^{\prime}$ and $|l|,|m| \leq N^{\prime}$, where the unspecified constant depends on $f$ but not on $l, m$ or $N^{\prime}$.

Proof. Follows directly from the aliasing properties of Fourier coefficients for smooth functions (cf. [44], Chap. 4).

Since one can choose $N^{\prime}$ in Lemma 5.5 proportional $^{1}$ to $N$, approximating the set of integrals $\left\{I_{l}^{1}\right\}_{l=-N}^{N}$ involves $O(2 N+1)$ evaluations of $f$ and one application of the FFT to the corresponding vector. Thus, the total $\operatorname{cost}^{2}$ is $O((2 N+1) \log (2 N+1))$ arithmetic operations - plus $O(2 N+1)$ function evaluations of $f$ - to approximate the $2 N+1$ integrals. The approximation of the set of integrals $\left\{I_{l, m}^{2}\right\}_{l, m=-N}^{N}$ requires $O\left((2 N+1)^{2}\right)$ evaluations of the function $F$ and $O(2(2 N+1))$ applications of the FFT to the corresponding vectors, yielding a cost of $O\left((2 N+1)^{2} \log (2 N+1)\right)$ arithmetic operations - plus $O\left((2 N+1)^{2}\right)$ function evaluations of $F$.

On the other hand, the block matrices $A_{i}$ in (4.18) consist of differences of self-interaction operators on $\Gamma_{i}$ for the four BIOs. While the difference of two operators is compact - the resulting kernel is smoother than that associated to a single evaluation of the same operator - the kernel is not arbitrarily smooth, even if the geometry is. Consequently, a deeper analysis is required before applying classical algorithms for the computation of Fourier transforms.

\footnotetext{
${ }^{1}$ The number of degrees of freedom is $2 N+1$.

${ }^{2}$ This is the classical estimation of the computational cost for the FFT.
} 
Let us consider, as an illustrative example, the weakly singular operator. We are required to compute integrals such as

$$
\int_{0}^{2 \pi} \int_{0}^{2 \pi} \widehat{G}_{\theta}^{k}(s, t) e^{-\imath l s} e^{\imath m t} \mathrm{~d} t \mathrm{~d} s
$$

where $\widehat{G}_{\theta}^{k}$ is as in (4.7). Decomposing $\widehat{G}_{\theta}^{k}$ as shown in (4.8), we obtain two integrals,

$$
I_{l, m}^{S}:=\int_{0}^{2 \pi} \int_{0}^{2 \pi} S(t-s) J_{\theta}^{k}(s, t) e^{-\imath l s} e^{\imath m t} \mathrm{~d} t \mathrm{~d} s, \quad I_{l, m}^{R}:=\int_{0}^{2 \pi} \int_{0}^{2 \pi} R_{\theta}^{k}(s, t) e^{-\imath l s} e^{\imath m t} \mathrm{~d} t \mathrm{~d} s .
$$

Since $R_{\theta}^{k}(s, t)$ is smooth and periodic (see Section 4.2.1), $I_{l, m}^{R}$ may be computed via the FFT. To compute $I_{l, m}^{S}$, we use the expansion ( $c f .[25]$, Eq. 12):

$$
S(t-s)=\sum_{\substack{n=-\infty \\ n \neq 0}}^{\infty} \frac{1}{4 \pi n} e^{\imath n(t-s)}
$$

Thus,

$$
I_{l, m}^{S}=\sum_{\substack{n=-\infty \\ n \neq 0}}^{\infty} \frac{1}{4 \pi n} \int_{0}^{2 \pi} \int_{0}^{2 \pi} J_{\theta}^{k}(s, t) e^{-\imath(l+n) s} e^{\imath(m+n) t} \mathrm{~d} t \mathrm{~d} s
$$

Since $J_{\theta}^{k}(s, t)$ is smooth and periodic $(c f .(4.10))$, each of the integrals of the right-hand side is easy to compute. Moreover, the terms in the series in (5.8) decay exponentially and the series may be truncated at the cost of a small approximation error. Furthermore, the sum in (5.8) may be understood as a discrete convolution, allowing it to be computed by multiplying the corresponding Fourier transforms (see [25] for details).

Lemma 5.6. For $N^{\prime}, \tilde{N} \in \mathbb{N}$, let $I_{l, m}^{S, N^{\prime}, \widetilde{N}}$ denote the approximation of $I_{l, m}^{S}$ computed through a $2 N^{\prime}+1$ point interpolation of $J_{\theta}^{k}(s, t)$ via FFT and approximating $S(t-s)$ by approximating the sum in (5.7) as

$$
\sum_{\substack{n=-\widetilde{N} \\ n \neq 0}}^{\widetilde{N}} \frac{1}{4 \pi n} e^{\imath n(t-s)}
$$

Then, it holds that

$$
\left|I_{l, m}^{S, N^{\prime}, \widetilde{N}}-I_{l, m}^{S}\right| \lesssim\left(N^{\prime}\right)^{-p} \log \widetilde{N}+\left(N^{\prime}+\widetilde{N}\right)^{-p}
$$

for all $l, m \in \mathbb{Z}$ and $p \in \mathbb{N}$ satisfying $p<N^{\prime}$ and $|l|,|m| \leq N^{\prime}$, where the unspecified constant depends only on the kernel $J_{\theta}^{k}(s, t)$.

Proof. Notice that

$$
\left|I_{l, m}^{S, N^{\prime}, \widetilde{N}}-I_{l, m}^{S}\right| \leq\left|\sum_{\substack{n=-\widetilde{N} \\ n \neq 0}}^{\widetilde{N}} \frac{1}{4 \pi n}\left(I_{l+n, m+n}^{J}-I_{l+n, m+n}^{J, N^{\prime}}\right)\right|+\left|\sum_{|n|>\widetilde{N}} \frac{1}{4 \pi n} I_{l+n, m+n}^{J}\right|,
$$

where

$$
I_{l+n, m+n}^{J}=\int_{0}^{2 \pi} \int_{0}^{2 \pi} J_{\theta}^{k}(s, t) e^{-\imath(l+n) s} e^{\imath(m+n) t} \mathrm{~d} t \mathrm{~d} s
$$

and $I_{l+n, m+n}^{J, N^{\prime}}$ is the approximation of $I_{l+n, m+n}^{J}$ obtained as in Lemma 5.5. The result then follows from Lemma 5.5 and the decay of Fourier coefficients of smooth functions ([44], Chap. 4). 
The computational cost of approximating $\left\{I_{l, m}^{S}\right\}_{l, m=-N}^{N}$ is dominated by the cost of the approximation of $\left\{I_{l, m}^{R}\right\}_{l, m=-N}^{N}$, since it involves $\left(2 N^{\prime}+1\right)^{2}$ evaluations of the quasi-periodic Green's function, each of which corresponds to $2 \widetilde{N}+1$ evaluations of a Hankel function ( $c f$. [14]). Choosing $N^{\prime}$ and $\widetilde{N}$ proportional to $N$ leaves the cost of evaluating the quasi-periodic Green's function as $O\left((2 N+1)^{3}\right)$ evaluations of the Hankel function ${ }^{3}$. Meanwhile, the total cost for $I_{l, m}^{S}$ is $O((2 N+1) \log (2 N+1))$.

For the operators $\mathrm{K}_{\theta}^{k}$ and $\mathrm{K}_{\theta}^{\prime \prime}$, a similar technique can be applied using (4.13). The integrals corresponding to the hyper-singular BIO are approximated by first using the integration-by-parts formula in Lemma 4.13, reducing it to two different integrals which are then approximated as those corresponding to the weakly singular BIO.

Considering $M>1$ interfaces, $2 N+1$ degrees of freedom on each interface and $N^{\prime}$ proportional to $N$ the total cost of the matrix assembly process can be estimated as $O\left(N^{3} M\right)$ Hankel function evaluations and $O\left(M N^{2} \log N\right)$ arithmetic operations. We point out that the cost could be reduced drastically by constructing an accurate algorithm to approximate the Hankel functions by pre-computing some values. We then have the following corollary of Theorem 5.2 and Lemmas 5.5 and 5.6.

Corollary 5.7. Let $\boldsymbol{N} \subset \mathbb{N}$ be as in Theorem 5.2 and consider the fully discrete problem, where we seek a solution $\widetilde{\mathbf{\Lambda}}^{N} \in \mathbb{E}_{\theta}^{N}$ of Problem 5.1, and consider the approximation of the discrete operator and right-hand side via FFT as in Lemmas 5.5 and 5.6. Then, the fully discrete problem is well posed and there exists $N^{\star} \in \mathbb{N}$ such that, for all $N^{\prime}, \widetilde{N} \geq N^{\star}$ defined as in Lemma 5.5 and 5.6, for which

$$
\left\|\boldsymbol{\Lambda}-\widetilde{\boldsymbol{\Lambda}}^{N}\right\|_{\mathcal{V}_{\theta}^{\frac{1}{2},-\frac{1}{2}}} \lesssim\left(\max _{i \in\{1, \ldots, M\}} N_{i}^{-s}\right)\|\varrho\|_{\mathcal{V}_{\theta}^{s_{1}, s_{2}}} .
$$

Proof. The result is an immediate consequence of Strang's lemma ([38], Sect. 4.2.4), using Lemma 5.5, and 5.6 to bound the quadrature errors.

\section{NUMERICAL EXAMPLES}

We now showcase computational experiments verifying the convergence estimates found in Theorem 5.2. The implementation of the aforementioned algorithms was carried through a $\mathrm{C}++$ cpu-only library. All the experiments ran on a Intel I7-4770@3.4GHZ processor with 8 threads. The code was compiled with gcc 4.9.4, openmp and $\mathrm{O} 2$ flags on. As in Section 5.3, we consider our discrete spaces with $2 N+1$ bases per interface, with $N \in \mathbb{N}$.

\subsection{Code validation}

We begin by considering the simple case of a grating with two media separated by a single horizontal line segment acting as its layer. Hence, using the following expansion of the Green's function ([5], Prop. 4.2):

$$
G_{\theta}^{k}(\boldsymbol{x}, \boldsymbol{y})=\frac{\imath}{4 \pi} \sum_{j \in \mathbb{Z}} \frac{1}{\beta_{j}} e^{\imath \beta_{j}\left|x_{2}-y_{2}\right|-\imath j_{\theta}\left(y_{1}-x_{1}\right)} \text { for all } \boldsymbol{x}, \boldsymbol{y} \in \mathbb{R}^{2},
$$

it is possible to assemble the matrix analytically. The matrix $\mathcal{M}$ is then composed of only block diagonal terms. Since the right-hand side only has two non-null components ${ }^{4}$, only the corresponding components for the solution are non-zero, yielding a closed form for the solution.

In order to test the implementation, we consider an artificial (harder) problem by including ghost domains, i.e., we add extra smooth (ghost) layers that separate domains with the same refraction index. Hence, the solution is the same as if these additional domains did not exist and has a closed form, as before. The results for different ghost layers are reported in Figure 3.

\footnotetext{
${ }^{3}$ The value of $\widetilde{N}$ has to be chosen depending of $k_{0}$, but typically one can assume that it need not be greater than $2 N$, for $N$ large enough to ensure convergence.

${ }^{4}$ One for the Dirichlet trace of the incident wave and another for the Neumann trace.
} 


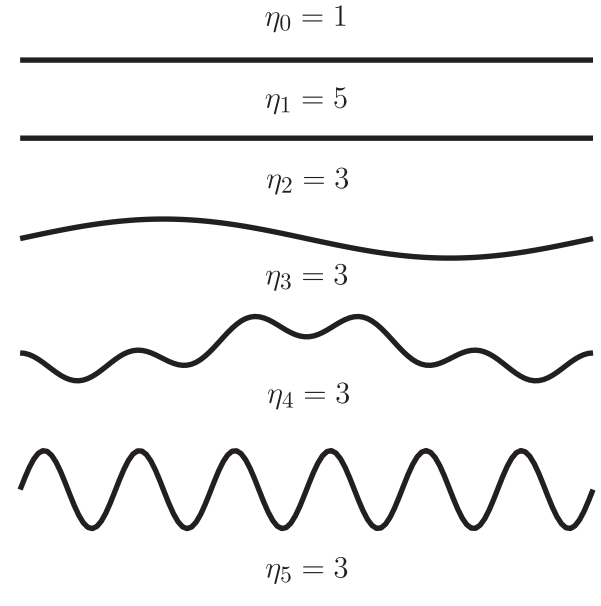

(a)

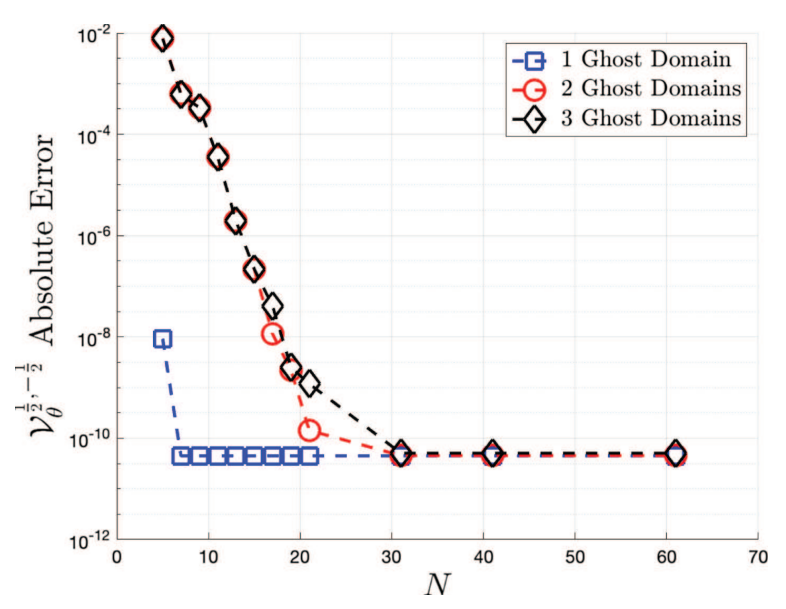

(b)

Figure 3. (a) Problem geometry. (b) Error in the $\mathcal{V}_{\theta}^{\frac{1}{2},-\frac{1}{2}}$ norm with respect to the analytic solution. Results provided for different numbers ghost layers (1,2 and 3 , respectively), i.e., the first experiment considers only the first 3 layers (counting downwards), the second one considers the first 4 layers and the third considers all 5 layers.

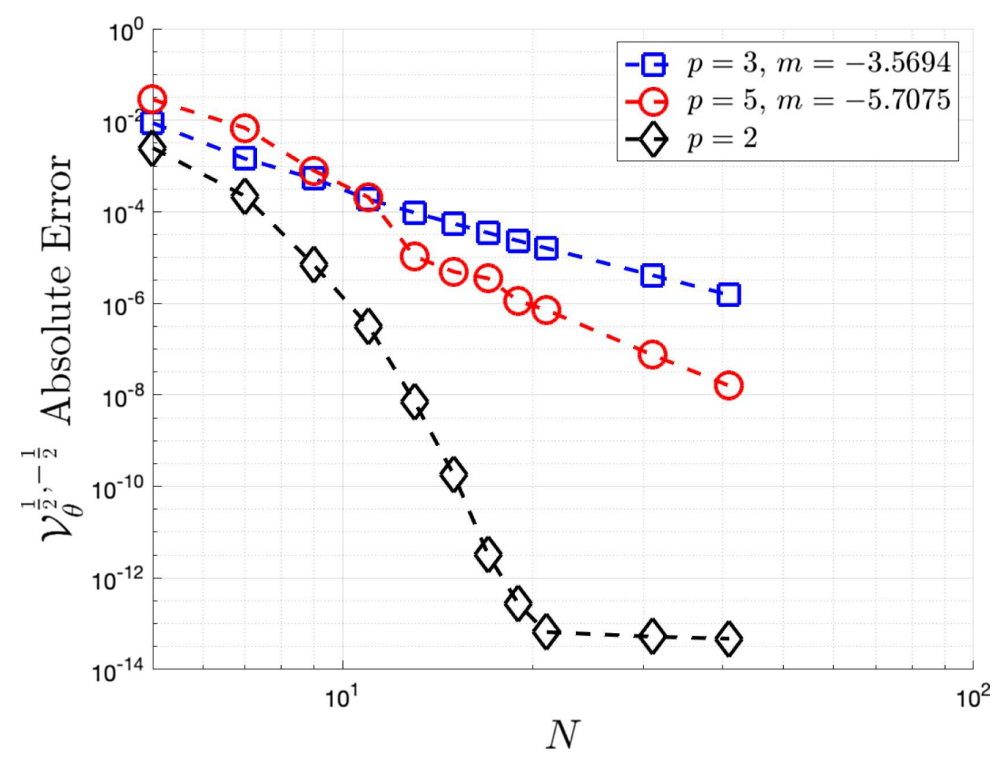

FigURE 4. Error in the $\mathcal{V}_{\theta}^{\frac{1}{2},-\frac{1}{2}}$ norm with respect to the analytic solution. The legend indicates an estimate of the slope of the error convergence curves for different values of $p$ (degrees of smoothness). Classically, error convergence estimates for spectral methods indicate the slope to be at least equal to $p$. We also consider the case $p=2$, where the extra layer is $\mathcal{C}^{\infty}$ and the super-algebraic convergence rate is observed. 
TABLE 1. Value of refraction indices $\left\{\eta_{i}^{(1)}\right\}_{i=1}^{12}$ and $\left\{\eta_{i}^{(2)}\right\}_{i=1}^{12}$ (corresponding to the two considered cases) for the grating in Figure 6 (counting downwards).

\begin{tabular}{l|llllllllllll}
\hline \hline & 1 & 2 & 3 & 4 & 5 & 6 & 7 & 8 & 9 & 10 & 11 & 12 \\
\hline$\eta_{i}^{(1)}$ & 4.7 & 4.2 & 4.8 & 3.6 & 1.1 & 4.4 & 4.7 & 3.7 & 4.0 & 3.9 & 2.6 & 3.6 \\
$\eta_{i}^{(2)}$ & 4.7 & 8.4 & 4.8 & 7.2 & 1.1 & 8.8 & 4.7 & 7.4 & 4.0 & 7.8 & 2.6 & 7.2 \\
\hline
\end{tabular}

We also display the convergence behaviour of the method for interfaces with limited regularity by repeating the previous experiment (same incident field) with one ghost domain and an interface given by

$$
\boldsymbol{z}_{3}(t)=\left(t, a|\sin (t)|^{p}+b\right)
$$

where $a, b$ are real numbers that scale the interface, and $p$ is an odd integer that determines the smoothness degree of the interface. In particular, $\boldsymbol{z}_{3}$ is in $\mathcal{C}^{p-2,1}$ or, more precisely, $\mathcal{C}^{p-1}$ with an integrable $p$-th derivative. Results are reported in Figure 4.

For all experiments in this section, the frequency is chosen as $k_{0}=1$ and the incidence angle is 0.47 radians.

\subsection{Convergence results}

We now consider a smooth geometry composed of 12 layers with varying refraction indices. Two different scenarios are employed, reported in Table $1, \eta_{i}^{(1)}$ and $\eta_{i}^{(2)}$, respectively. We also consider three different wavenumbers for the incident wave, $k_{0}=2.8,14$ and 28. Convergence results in the energy norm for the solution of Problem 5.1 for the different cases of parameters and wavenumbers are reported in Figure 5, where exponential convergence is observed for all considered scenarios, as expected. All errors were computed with respect to an overkill solution, with approximately 50 more bases per interface than the last plotted point for each curve. The incidence angle is, again, 0.47 radians.

Finally, in Figure 6 we present an illustration of the total field corresponding to the refraction indices given in Table 1 for case 1 . The fields were obtained by using enough degrees of freedom so as to ensure an error of order $10^{-2}$ in the energy norm, i.e., $N \approx 40,130$ and 250 for $k_{0}=2.8,14$ and 28 , respectively.

Remark 6.1. Though establishing the relation between the parameters $-\left\{\eta_{i}\right\}_{i=0}^{M}$ and $k_{0}$ - and the number of basis elements required to attain a certain desired accuracy is not straightforward, our experiments suggest that $N$ should be chosen proportional to the maximum wavenumber $k_{\max }:=\max _{i \in\{0, \ldots M\}} k_{i}$.

\section{Conclusions}

We have proposed a fast spectral method for the efficient representation, through surface potentials based on the quasi-periodic Green's function, for the solution of the Helmholtz equation with transmission boundary conditions on a periodic domain. In Theorem 5.2, we obtained convergence estimates for the discrete approximation of the corresponding boundary data, and found that discrete solutions converge at a super-algebraic rate to continuous solutions of the considered BIE. Though we focused on the Helmholtz transmission problem, our approximation results and convergence estimates can be easily extended to other BIEs on quasi-periodic Sobolev spaces whenever the formulation is well posed. We avoided cut-off frequencies from our analysis since 


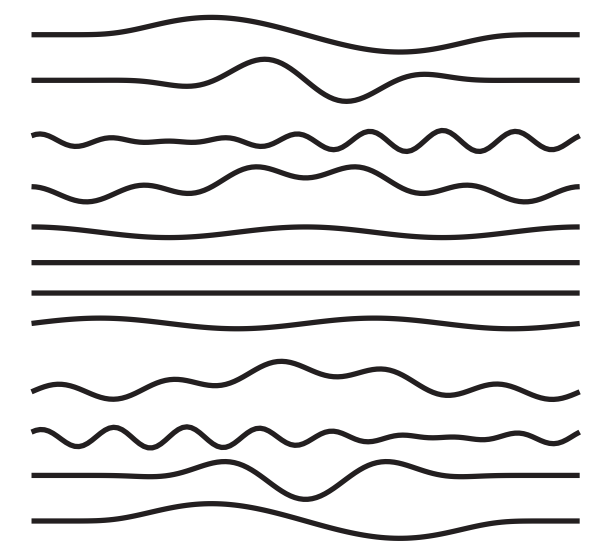

(a)

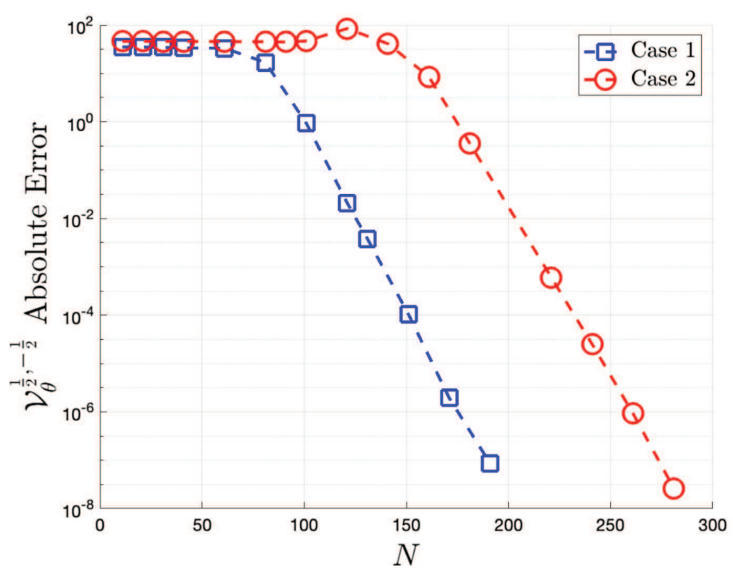

(c)

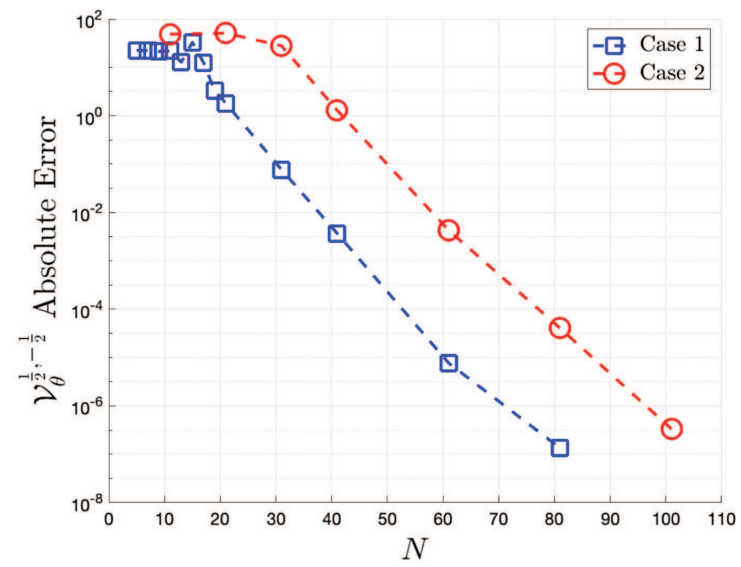

(b)

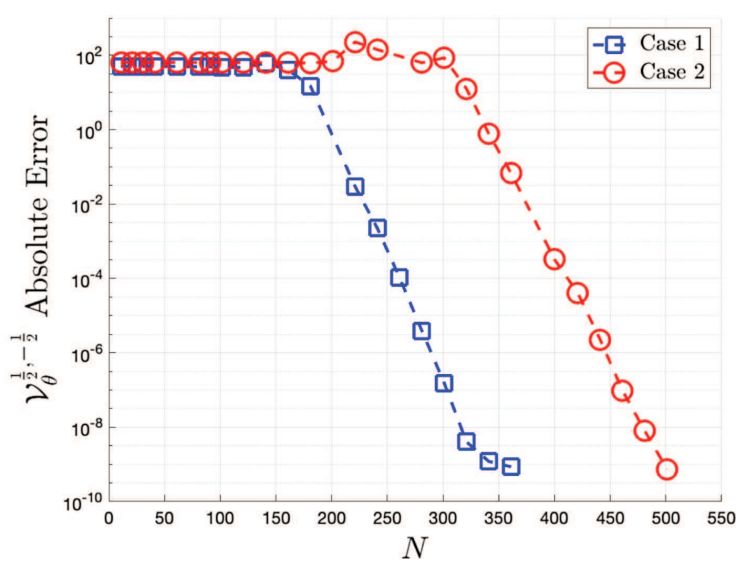

(d)

Figure 5. (a) Problem geometry with 12 layers. (b), (c) and (d) Errors in the corresponding energy norm for $k_{0}=2.8,14.0,28.0$. Each of these subfigures present error convergence curves for the two scenarios of refraction indices considered and specified in Table 1. Notice that the curves in red - corresponding to parameters $\eta_{i}^{(2)}$ in Table 1 - display a longer preasymptotic regime before convergence is observed for all considered values of $k_{0}$, seemingly due the presence of layers with higher wavenumbers (see Rem. 6.1).

the series in (4.2) fails to converge for said frequencies and, for the same reason, our previous results from [5] exclude them as well.

Despite similar numerical results are known for the Nyström Method, theoretical grounds for the observed convergence rates are scarce [12]. In contrast, the Galerkin discretizations presented in this article lead to provable convergence rates equal to those expected by Nyström methods.

Future work considers: (i) including cut-off frequencies in our analysis, (ii) extending our results to three dimensional Helmholtz equations and Maxwell's equations on periodic domains and (iii) applications in uncertainty quantification [40] and shape optimization [6]. 


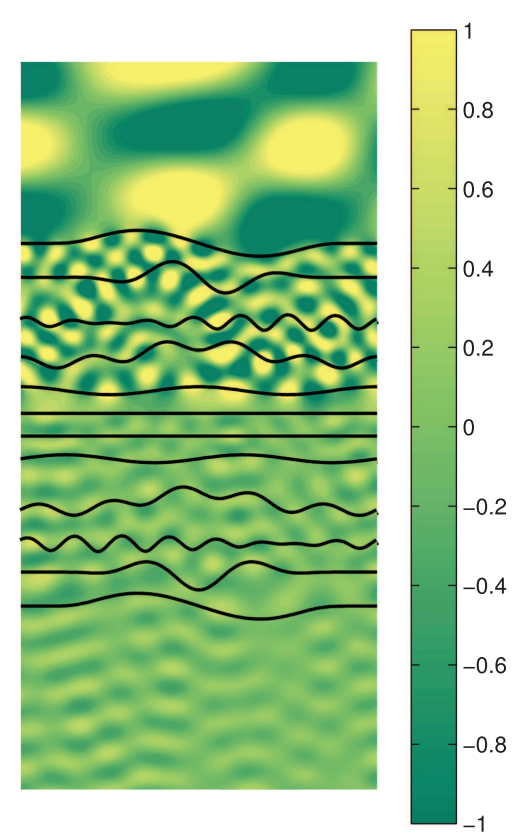

(a)

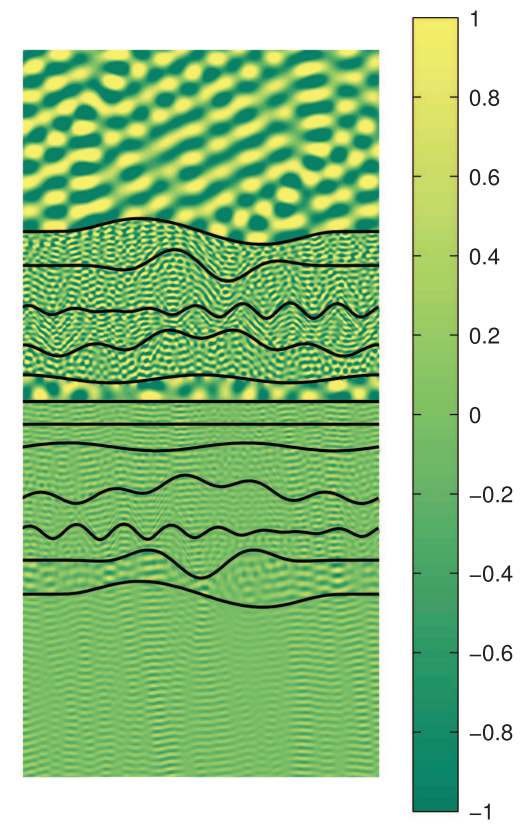

(b)

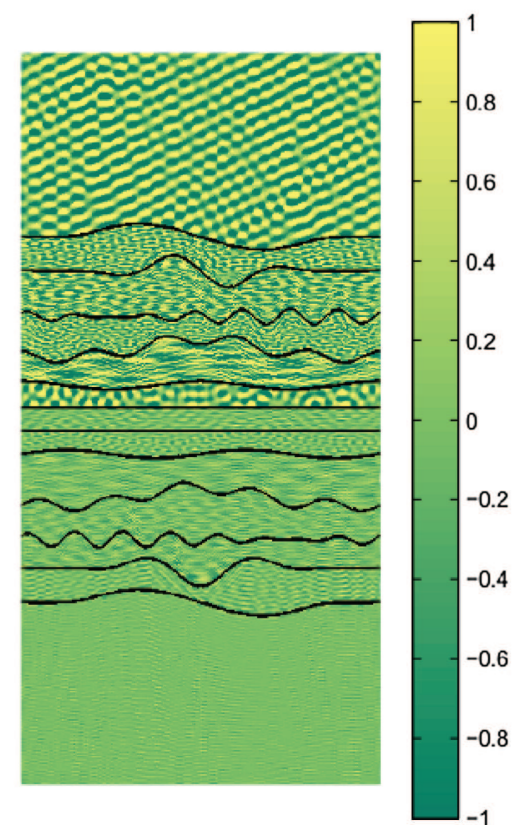

(c)

Figure 6. Real part of the total wave $\left(u^{(\text {tot })}=u^{(\mathrm{sc})}+u^{(\mathrm{inc})}\right)$ for each different value of $k_{0}$, namely $2.8,14$ and 28 . The refraction indices on each layer are those indicated on Table 1 . The incidence angle is again 0.47. (a) $k_{0}=2: 8$. (b) $k_{0}=14$. (c) $k_{0}=28$. 
Acknowledgements. This work was partially funded by Fondecyt Regular 1171491 and by grants ConicytPFCHA/Doctorado Nacional/2017-21171791 and 2017-21171479.

\section{REFERENCES}

[1] M. Abramowitz and I.A. Stegun, Handbook of Mathematical Functions: with Formulas, Graphs, and Mathematical Tables. In: Vol. 55 Courier Corporation (1965).

[2] H. Ammari, Scattering of waves by thin periodic layers at high frequencies using the on-surface radiation condition method. IMA J. Appl. Math. 60 (1998) 199-214.

[3] H. Ammari and J.C. Nédélec, Analysis of the Diffraction from Chiral Gratings. In: Mathematical Modeling in Optical Science. SIAM (2001) 179-206.

[4] H. Ammari and G. Bao, Coupling of finite element and boundary element methods for the scattering by periodic chiral structures. J. Comput. Math. 26 (2008) 261-283.

[5] R. Aylwin, C. Jerez-Hanckes and J. Pinto, On the properties of quasi-periodic boundary integral operators for the Helmholtz equation. Integral Equ. Oper. Theory 92 (2020) 17.

[6] R. Aylwin, G. Silva-Oelker, C. Jerez-Hanckes and P. Fay, Optimization methods for achieving high diffraction efficiency with perfect electric conducting gratings. J. Opt. Soc. Am. A 37 (2020) 1316-1326.

[7] G. Bao, Variational approximation of Maxwell's equations in biperiodic structures. SIAM J. Appl. Math. 57 (1997) 364-381.

[8] G. Bao, Recent mathematical studies in the modelling of optics and electromagnetics. J. Comput. Appl. Math. 22 (2004) $148-155$.

[9] G. Bao and D.C. Dobson, On the scattering by a biperiodic structure. Proc. Am. Math. Soc. 128 (2000) $2715-2723$.

[10] G. Bao, D.C. Dobson and J.A. Cox, Mathematical studies in rigorous grating theory. J. Opt. Soc. Am. A 12 (1995) $1029-1042$.

[11] A. Barnett and L. Greengard, A new integral representation for quasi-periodic scattering problems in two dimensions. BIT Numer. Math. 51 (2011) 67-90.

[12] Y. Boubendir, V. Dominguez and C. Turc, High-order Nyström discretizations for the solution of integral equation formulations of two-dimensional Helmholtz transmission problems. IMA J. Numer. Anal. 36 (2014).

[13] O.P. Bruno and M.C. Haslam Efficient high-order evaluation of scattering by periodic surfaces: deep gratings, high frequencies, and glancing incidences. JOSA A 26 (2009) 658-668.

[14] O.P. Bruno and B. Delourme Rapidly convergent two-dimensional quasi-periodic Green's function throughout the spectrum including Wood anomalies. J. Comput. Phys. 262 (2014) 262-290.

[15] O.P. Bruno and A.G. Fernandez-Lado, Rapidly convergent quasi-periodic Green's functions for scattering by arrays of cylinders - including Wood anomalies. Proc. R. Soc. A 473 (2017) 20160802.

[16] O.P. Bruno, S.P. Shipman, C. Turc and S. Venakides, Superalgebraically convergent smoothly windowed lattice sums for doubly periodic green functions in three-dimensional space. Proc. R. Soc. A 472 (2016) 20160255.

[17] O.P. Bruno, S.P. Shipman, C. Turc and V. Stephanos Three-dimensional quasi-periodic shifted Green's function throughout the spectrum, including Wood anomalies. Proc. R. Soc. A 473 (2017) 20170242.

[18] Y.B. Chen and Z. Zhang, Design of tungsten complex gratings for thermophotovoltaic radiators. Opt. Commun. 269 (2007) $411-417$.

[19] M.H. Cho and A.H. Barnett, Robust fast direct integral equation solver for quasi-periodic scattering problems with a large number of layers. Opt. Express 23 (2015) 1775-1799.

[20] D. Colton and R. Kress, Integral Equation Methods in Scattering Theory. Society for Industrial and Applied Mathematics, Philadelphia, PA (2013)

[21] D.C. Dobson and A. Friedman, The time-harmonic Maxwell equations in a doubly periodic structure. J. Math. Anal. Appl. 166 (1992), 507-528.

[22] J. Elschner and G. Schmidt, Diffraction in periodic structures and optimal design of binary gratings. Part i: direct problems and gradient formulas. Math. Methods Appl. Sci. 21 (1998) 1297-1342.

[23] I.G. Graham and I.H. Sloan, Fully discrete spectral boundary integral methods for Helmholtz problems on smooth closed surfaces in $\mathbb{R}^{3}$. Numer. Math. 92 (2002) 289-323.

[24] L. Greengard, K.L. Ho and J.Y. Lee, A fast direct solver for scattering from periodic structures with multiple material interfaces in two dimensions. J. Comput. Phys. 258 (2014) 738-751.

[25] F.Q. Hu, A spectral boundary integral equation method for the 2D Helmholtz equation. J. Comput. Phys. 120 (1995) 340-347.

[26] C. Jerez-Hanckes and J. Pinto, High-order Galerkin method for Helmholtz and Laplace problems on multiple open arcs. ESAIM: M2AN 54 (2020) 975-2009.

[27] A. Kirsch, Diffraction by Periodic structures. In: Inverse Problems in Mathematical Physics. Springer (1993) 87-102.

[28] R. Kress, Linear Integral Equations, 3rd edition. In: vol. 82 Applied Mathematical Sciences (2014).

[29] C.M. Linton, The Green's function for the two-dimensional Helmholtz equation in periodic domains. J. Eng. Math. 33 (1998) $377-401$.

[30] Y. Liu and A. Barnett, Efficient numerical solution of acoustic scattering from doubly-periodic arrays of axisymmetric objects. J. Comput. Phys. 324 (2016) 226-245.

[31] E.G. Loewen and E. Popov, Diffraction Gratings and Applications. CRC Press (2018).

[32] W.C.H. McLean, Strongly Elliptic Systems and Boundary Integral Equations. Cambridge University Press (2000). 
[33] Y. Nakata and M. Koshiba, Boundary-element analysis of plane-wave diffraction from groove-type dielectric and metallic gratings. JOSA A $\mathbf{7}$ (1990) 1494-1502.

[34] J.C. Nédélec and F. Starling, Integral equation methods in a quasi-periodic diffraction problem for the time-harmonic Maxwell's equations. SIAM J. Math. Anal. 22 (1991) 1679-1701.

[35] D. Nguyen, Spectral methods for direct and inverse scattering from periodic structures, Ph.D. thesis, École Polytechnique (2012).

[36] E. Popov, Gratings: Theory and Numeric Applications. Popov, Institut Fresnel (2012).

[37] J. Saranen and G. Vainikko, Periodic Integral and Pseudodifferential Equations with Numerical Approximation. Springer Science \& Business Media (2013).

[38] S.A. Sauter and C. Schwab, Boundary Element Methods. In: Vol. 39 Springer Series in Computational Mathematics (2011).

[39] G. Silva, C. Jerez-Hanckes and P. Fay, High-temperature tungsten-hafnia optimized selective thermal emitters for thermophotovoltaic applications. J. Quant. Spectrosc. Radiat. Transf. 231 (2019) 61-68.

[40] G. Silva-Oelker, R. Aylwin, C. Jerez-Hanckes and P. Fay, Quantifying the impact of random surface perturbations on reflective gratings. IEEE Trans. Antennas Propag. 66 (2018) 838-847.

[41] F. Starling and A.S. Bonnet-Bendhia, Guided waves by electromagnetic gratings and non-uniqueness examples for the diffraction problem. Math. Methods Appl. Sci. 17 (1994) 305-338.

[42] O. Steinbach, Numerical Approximation Methods for Elliptic Boundary Value Problems. Springer Science \& Business Media (2007).

[43] M. Taibleson, Fourier coefficients of functions of bounded variation. In: Vol. 18 Proc. Amer. Math. Soc. (1967).

[44] L.N. Trefethen, Spectral Methods in Matlab. In: Society for Industrial and Applied Mathematics. USA (2000).

[45] B. Zhang and S.N. Chandler-Wilde, A uniqueness result for scattering by infinite rough surfaces. SIAM J. Appl. Math. 58 (1998) 1774-1790.

[46] Y. Zhang and A. Gillman, A fast direct solver for two dimensional quasi-periodic multilayered medium scattering problems. Preprint arXiv: 1907.06223 (2019).

\section{Subscribe to Open (S2O) A fair and sustainable open access model}

This journal is currently published in open access under a Subscribe-to-Open model (S2O). S2O is a transformative model that aims to move subscription journals to open access. Open access is the free, immediate, online availability of research articles combined with the rights to use these articles fully in the digital environment. We are thankful to our subscribers and sponsors for making it possible to publish this journal in open access, free of charge for authors.

\section{Please help to maintain this journal in open access!}

Check that your library subscribes to the journal, or make a personal donation to the S2O programme, by contacting subscribers@edpsciences.org

More information, including a list of sponsors and a financial transparency report, available at: https://www. edpsciences.org/en/maths-s2o-programme 